\title{
Desenvolvimento e Avaliação de Calorímetros por Nitrogênio Líquido e Fluxo Contínuo para Medição de Aporte Térmico
}

\author{
(Development and Assessment of Calorimeters Using Liquid Nitrogen and Continuous Flow (Water) for Heat Input \\ Measurement)
}

\author{
Hernán Dario Hernández Arevalo ${ }^{1}$, Louriel Oliveira Vilarinho ${ }^{1}$ \\ ${ }^{1}$ Laprosolda - Centro para Pesquisa e Desenvolvimento de Processos de Soldagem, Universidade Federal de Uberlândia, \\ Uberlândia/MG,Brasil,vilarinho@mecanica.ufu.br
}

\begin{abstract}
Resumo
Um dos parâmetros mais influentes no processo de soldagem a nível industrial e de pesquisa é o calor entregue à chapa (aporte térmico) devido a sua direta ligação com a mudança das características metalúrgicas e propriedades mecânicas da junta soldada. Para o estudo e quantificação do calor aportado na peça de trabalho, tem-se utilizado tanto métodos teóricos (analíticos e numéricos), quanto experimentais (calorimetria). Estas técnicas apresentam ampla dispersão dos resultados obtidos para o rendimento térmico. Desta forma, a proposta deste trabalho é apresentar o projeto, construção e avaliação de um calorímetro de nitrogênio líquido e fluxo contínuo (água) para a medição do aporte térmico e rendimento térmico de processos GTAW e GMAW. Para o processo GTAW, cordões sobre chapa foram realizados com Ar puro e chapas de aço ao carbono ASTM A36 e variou-se os parâmetros de soldagem como corrente, comprimento do arco, velocidade de soldagem e comprimento do cordão de solda. Foram também realizadas soldagens sobre chapa com o processo GMAW em transferência por curto-circuito e goticular (spray), onde o arame utilizado foi AWS ER70S-6 (1,2 mm de diâmetro)e gás de proteção Ar+25\%CO2 (curto-circuito) e Ar+5\%O2 (goticular). Para os processos derivativos (STT - Surface Tension Transfer, RMD - Regulated Metal Deposition, CMT - Cold Metal Transfer, GMAW-P - Pulsado e GMAW-PV-Polaridade Variável) foram tomados como base parâmetro de soldagem encontrados por outros autores no Laprosolda (Centro para Pesquisa e Desenvolvimento de Processos de Soldagem). De modo geral, o calorímetro de nitrogênio líquido apresentou boa repetibilidade com diferença máxima de 3\%. Também foi observado que durante os testes realizados no calorímetro de nitrogênio liquido apresentaram resultados coerentes para as diferentes condições de soldagem e reportando valores de rendimento térmico global de 70,3\% para o processo GTAW, 78,6\% para GMAW curto-circuito convencional, 73,6\% para GMAW goticular e 76,1\% para os processos GMAW curto circuito-controlado. Os resultados obtidos com o calorímetro de fluxo contínuo (água) acompanharam a tendência do calorímetro de nitrogênio líquido, mas com resultados médios $12 \%$ abaixo.
\end{abstract}

Palavras-chave: Aporte térmico, Rendimento térmico, Calorimetria, Nitrogênio Líquido, Fluxo contínuo, Processos Derivativos.

\begin{abstract}
One of the most influent parameter on the welding process at both industrial and research levels is the heat delivered to the workpiece (heat input) due to its direct connection with changes in metallurgical characteristics and mechanical properties of the weld joint. In order to quantify the heat input, different methods have been developed, both theoretical (analytical and numerical ones) and experimental (calorimetry). These techniques present large dispersion of results for thermal efficiency of welding processes. Therefore, this work aims to present the project, construction and assessment of a liquid nitrogen calorimeter and other one based on constant flow (water) measurement. Both calorimeters were evaluated for measuring heat input and thermal efficiency of GTAW and GMAW processes. For GTAW process, bead on plate welding was carried out with Ar as shielding gas over ASTM A36 plate, varying current, arc length, travel speed and bead length. For GMAW processes, both short-circuit and spray transfers were used with AWS ER70S-6 wire with $1.2 \mathrm{~mm}$ of diameter and Ar+25\%CO2 (short-circuit) and Ar+5\%O2 (spray) as shielding gases. For derivative processes (STT - Surface Tension Transfer, RMD - Regulated Metal Deposition, CMT - Cold Metal Transfer, GMAW-P - Pulsed and GMAW$V P$ - Variable Polarity), the welding parameters were adopted from previous researches from Laprosolda (Center for Research and Development of Welding Processes). It can be concluded that the liquid nitrogen calorimeter presents good repeatability and stability (maximum data scattering of 3\%). Also, the obtained results are coherent for different welding conditions applied through the work with global thermal efficiencies of 70,3\% for GTAW, 78,6\% for conventional short-circuit GMAW, 73,6\% for GMAW with spray transfer and $76,1 \%$ for GMAW derivative processes. The results measured by the continuous flow (water) calorimeter followed the trend of the liquid nitrogen one, but with average results $12 \%$ lower.
\end{abstract}

Key-words: Thermal efficiency, Calorimetry, Liquid Nitrogen, Continuous Flow, Derivative Processes.

(Convidado em 15/05/2012; Texto final em 26/08/2012). 


\section{Introdução}

O estudo de aporte térmico em processos de soldagem tem implicado no desenvolvimento de diversos métodos para sua quantificação, tanto métodos analíticos, quanto experimentais, que foram evoluindo de acordo com o avanço tecnológico da época. Rosenthal [1] propõe um dos primeiros modelos analíticos para o estudo do fluxo de calor em duas e três dimensões realizando uma série de simplificações permitindo a solução do equacionamento. Partindo deste modelo, diferentes autores analisaram o fluxo de calor e rendimento térmico baseados nas medições dos ciclos térmicos, mas ainda com restrições nas propriedades do material e perdas de calor por convecção, radiação e condução [2]. Mais recentemente [3], os avanços tecnológicos, especificamente em computadores, foram essenciais para o desenvolvimento de modelos mais adequados do fenômeno de transferência de calor. Por isto a estimação do rendimento térmico através deste método não abrange todos os fatores associados a um sistema complexo de fluxo de calor em soldagem, sendo limitado por fatores associados ao próprio modelo e propriedades do material que podem afetar a solução do mesmo. Neste contexto, a solução experimental via calorimetria têm bons resultados, pois envolvem todas as variáveis que estão associadas a um sistema complexo de soldagem.

Os calorímetros utilizados no estudo térmico de processos de soldagem compreendem uma ampla gama de tipos que foram desenvolvidos de acordo com o avanço tecnológico e disponibilidade de materiais para sua construção e uso. Entre estes estão, o calorímetro de água [4] que é baseado no monitoramento da temperatura da água, o calorímetro que funciona baixo o principio Seebeck [5], o calorímetro de caixa isolada baseado na medição da temperatura de corpos com propriedades térmicas conhecidas e definidas [6] e o calorímetro de nitrogênio líquido $\left(\mathrm{N}_{2} \mathrm{~L}\right)$ que quantifica o aporte térmico através da massa evaporada de $\mathrm{N}_{2} \mathrm{~L}$ obtendo-se bons resultados $[7,8]$.

Desta forma, o objetivo principal deste trabalho é apresentar o projeto e fabricação do calorímetro de $\mathrm{N}_{2} \mathrm{~L}$, tendo como ponto de referência os trabalhos já realizados por outros autores, procurando atribuir uma melhora significativa no modo operativo do processo de medição, uma vez que está diretamente influenciado por parâmetros como o ambiente, pontos de contato da placa de teste, tempo de soldagem, tempo de transferência e intervenção humana. Tal dependência faz com que os testes tenham uma variação significativa de resultados devido à soma de cada um destes parâmetros levando à grande dispersão de resultados encontrada na literatura.

\section{Considerações do projeto}

$\mathrm{O}$ calorímetro de $\mathrm{N}_{2} \mathrm{~L}$ é utilizado para quantificar a energia absorvida pela chapa num processo de soldagem. O calor absorvido pela chapa durante a formação do cordão de solda é transferido para o $\mathrm{N}_{2} \mathrm{~L}$ levando-o a ponto de ebulição. A energia associada à mudança de fase de liquido a vapor do $\mathrm{N}_{2} \mathrm{~L}$ pode ser avaliada como a energia perdida pela chapa. A análise calorimétrica está baseada na perdida de massa do $\mathrm{N}_{2} \mathrm{~L}$. Com o conhecimento da massa evaporada e o calor latente de vaporização do $\mathrm{N}_{2} \mathrm{~L}$ pode-se determinar a quantidade de calor trocado, e consequentemente a quantidade de calor que está entrando na chapa devido ao processo de soldagem, expressada pela Eq. (1).

$\mathrm{Q}_{\text {Latente }}:=\mathrm{m}^{*} \mathrm{~L}$

Onde, $\mathrm{Q}_{\text {Latente }}$ : Quantidade de energia necessária para obter a mudança do $\mathrm{N}_{2}$ líquido [J]; m: Massa evaporada ou perdida do $\mathrm{N}_{2}$ líquido [g]; L: Calor latente de vaporização do $\mathrm{N}_{2} \mathrm{~L}[\mathrm{~kJ} / \mathrm{kg}]$.

$\mathrm{O} \mathrm{N}_{2} \mathrm{~L}$ é um gás liquefeito o seja está a temperaturas criogênicas (menores que $-150^{\circ} \mathrm{C}$ ). Esta característica faz que seu ponto de ebulição seja muito baixo ao redor de $-196^{\circ} \mathrm{C}$. $\mathrm{O}$ cálculo de calor absorvido, representado pela Eq. (2) está dividido em duas partes, um é o calor que contem a chapa depois do processo de soldagem e o outro é o calor que contem a chapa a temperatura ambiente.

$\mathrm{Q}_{\text {Absorvido }}=\Delta \mathrm{Q}_{\mathrm{PS} \rightarrow \mathrm{TN}_{2}}-\Delta \mathrm{Q}_{\mathrm{TA} \rightarrow \mathrm{TN}_{2}}$

Onde, $\mathrm{Q}_{\mathrm{Absorvido}}$ : Calor líquido absorvido pelo processo de soldagem $[\mathrm{J}] ; \Delta \mathrm{Q}_{\mathrm{PS} \rightarrow \mathrm{TN} 2}$ : Calor absorvido pelo $\mathrm{N}_{2} \mathrm{~L}$, com chapa na temperatura do processo de soldagem $[\mathrm{J}] ; \Delta \mathrm{Q}_{\mathrm{TA} \rightarrow \mathrm{TN} 2}$ : Calor absorvido pelo $\mathrm{N}_{2} \mathrm{~L}$, com chapa na temperatura ambiente $[\mathrm{J}]$.

O cálculo das parcelas de calor devido ao calor que contem a placa teste a temperatura ambiente e o calor imposto pelo processo de soldagem, Eq. (3) e (4), é obtido pela diferença de massa perdida de $\mathrm{N}_{2} \mathrm{~L}$ :

$\Delta \mathrm{Q}_{\mathrm{PS} \rightarrow \mathrm{TN}_{2}}=\left(\mathrm{m}_{\text {final }}-\mathrm{m}_{\text {inicial }}-\mathrm{m}_{\text {chapa }}\right) * \mathrm{~L}$

$\Delta \mathrm{Q}_{\mathrm{TA} \rightarrow \mathrm{TN}_{2}}=\left(\mathrm{m}_{\text {final }}-\mathrm{m}_{\text {inicial }}-\mathrm{m}_{\text {chapa }}\right) * \mathrm{~L}$

Onde, ; $\mathrm{m}_{\text {inicial }}$ : massa inicial registrada desde o ponto de estabilização do $\mathrm{N}_{2} \mathrm{~L}$ [g]; $\mathrm{m}_{\text {chapa }}$ : massa da chapa teste [g]; L: Calor latente de vaporização do $\mathrm{N}_{2} \mathrm{~L}[\mathrm{~kJ} / \mathrm{kg}]$.

Para se medir o calor de entrada num processo de soldagem, associado à massa perdida de $\mathrm{N}_{2} \mathrm{~L}$, tem-se utilizado diferentes configurações deste tipo de calorímetro [8-11], que fazem uso de uma configuração básica formada por um recipiente Dewar (que é um vaso projetado para proporcionar isolamento térmico, diminuindo as perdas de calor por condução, convecção e radiação), uma balança com saída de sinal, sistema de aquisição de dados para registrar os dados de perda de peso da balança, estrutura de proteção do sistema eumrecipiente de armazenamento de $\mathrm{N}_{2} \mathrm{~L}$ de alta capacidade, alem destes equipamentos se conta com os elementos de segurança necessários para a manipulação do $\mathrm{N}_{2} \mathrm{~L}$. O esquema da configuração geral está representado na Fig. (1). 


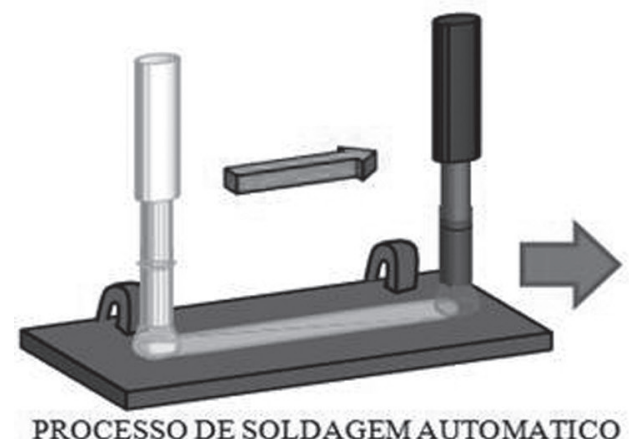

PROCESSO DE SOLDAGEM AUTOMATICO

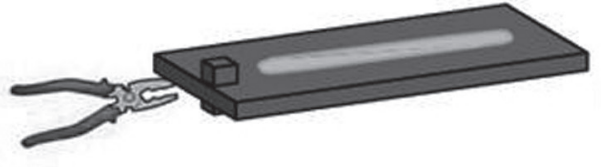

MOVIMENTAÇÃO MANUAL DA CHAPA

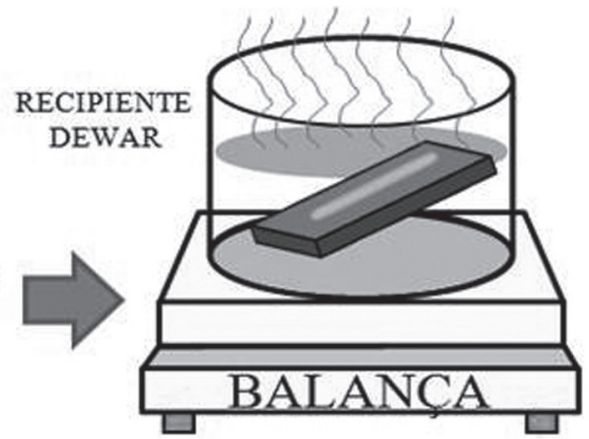

SISTEMA DE MEDIÇÃO DO NITROGENIO LIOUIDO EVAPORADO

Figura 1. Esquema geral do sistema de medição de massa perdida de $\mathrm{N}_{2} \mathrm{~L}$

O processo da formação do cordão de solda é feito automaticamente com uma mesa automática ou um robô para a movimentação da tocha de soldagem, assegurando um movimento lineal, uma constante distância bico de contato-peça e velocidade de soldagem. A chapa é fixada por um sistema que permite uma menor área de contato para diminuir as perdas de calor por condução, e ao mesmo tempo permita a rápida e fácil liberação da chapa para ser transferida ao recipiente Dewar.

Imediatamente depois da formação do cordão de solda, a chapa é liberada da fixação e transferida para o calorímetro de $\mathrm{N}_{2} \mathrm{~L}$, onde vai ser medida a massa perdida devido ao calor imposto no processo de soldagem deixando que a $\mathrm{N}_{2} \mathrm{~L}$ alcance a taxa de vaporização normal devido à temperatura ambiente. A seguir deve-se fazer uma nova medição do calor que contem a chapa a temperatura até que alcance de novo a taxa de vaporação normal. Com os dados adquiridos de massa perdida pode-se prosseguir a fazer os respectivos cálculos de eficiência térmica e calor imposto como foi explicado previamente com as Eq. (1) a (4).

Como considerações específicas vale ressaltar que o calor imposto em processos de soldagem é um fator muito sensível a mudanças de parâmetros operativos do processo de soldagem e parâmetros operativos diretamente envolvidos ao processo de medição, estas possíveis fontes de erro são:

- Material para fixar a placa teste durante o processo de soldagem;

- Tempo de formação do cordão (comprimento do cordão de solda);

- Movimento de transferência da placa teste ao calorímetro de $\mathrm{N}_{2} \mathrm{~L}$;

- Intervalo de tempo entre o final da solda ate o começo da medição de perda de peso pelo calorímetro;

- Entorno (umidade relativa, condições ambientais e presença de ventos durante o processo).

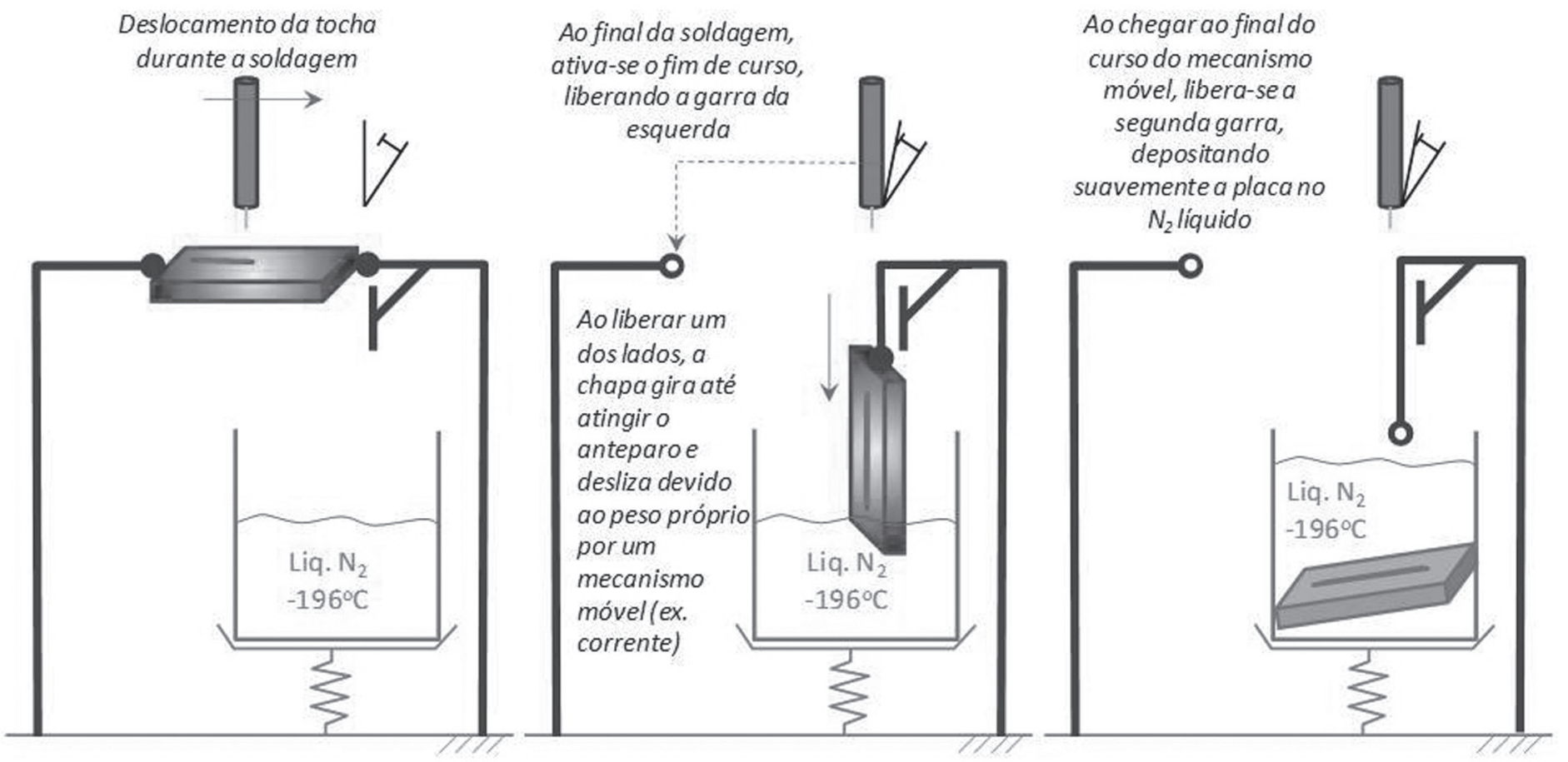

Figura 2. Esquema mecânico da bancada experimental para o calorímetro de $\mathrm{N}_{2}$ líquido 


\section{Projeto do calorímetro}

A maioria dos erros experimentais listados previamente tem a intervenção do operador sendo este um dos principais fatores que influi diretamente na medição do calor imposto. Assim, a proposta do trabalho é padronizar todos os movimentos e tempos que influem na operação e aquisição de dados do calorímetro de $\mathrm{N}_{2} \mathrm{~L}$, por meio de uma bancada experimental automatizada, conforme mostrado na Fig. (2).

$\mathrm{O}$ desenho e projeto da bancada experimental foram desenvolvidos com sistema CAD, permitindo analisar e simular os movimentos que estão envolvidos na manipulação da chapa desde o começo da solda até sua total imersão no $\mathrm{N}_{2} \mathrm{~L}$. A bancada experimental (Fig. 3) é composta por uma estrutura geral, sistema de manipulação da placa teste (garra fixa e garra móvel), sistema de manipulação da tocha de soldagem, sistema de medição de massa perdida de $\mathrm{N}_{2} \mathrm{~L}$, sistema pneumático para automação da bancada e sistema de aquisição de dados.

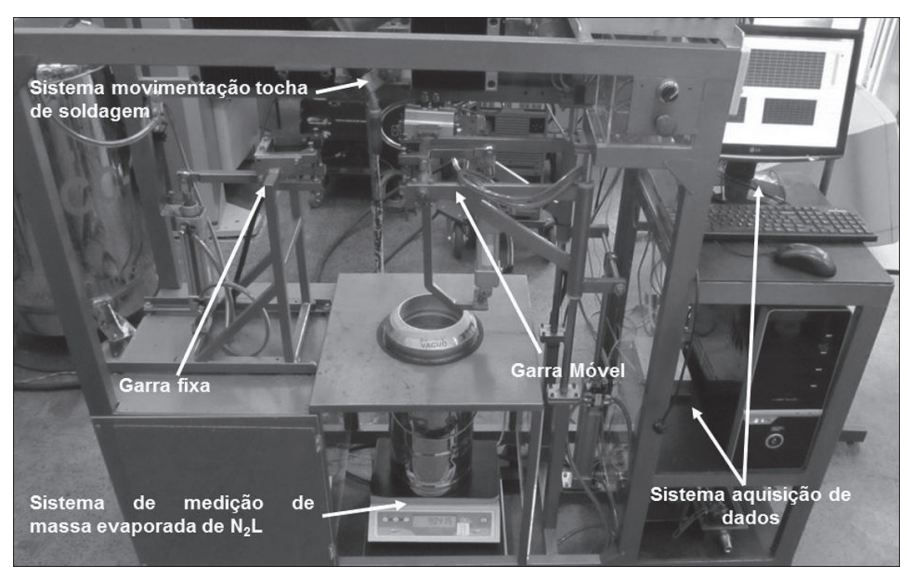

Figura 3. Montagem da bancada experimental de medição de aporte térmico com o calorímetro de $\mathrm{N}_{2} \mathrm{~L}$

\section{Avaliação do calorímetro}

\subsection{Avaliação do funcionamento do funcionamento do calorímetro com placas teste em condições ambientais}

Para verificar a repetibilidade do sistema se realizaram testes onde foram submersas placas testes no $\mathrm{N}_{2} \mathrm{~L}$ com diferentes espessuras, como é apresentado na Tab. 1. Realizando duas réplicas para cada espessura com o objetivo de ter resultados mais confiáveis de amostragem.

Tabela 1. Dimensões e massa das placas utilizadas para o teste repetibilidade

\begin{tabular}{|c|c|c|c|c|}
\hline Placa teste & Material & $\begin{array}{c}\text { Espessura } \\
{[\mathrm{mm}]}\end{array}$ & $\begin{array}{c}\text { Dimensões } \\
{[\mathrm{mm}]}\end{array}$ & $\begin{array}{c}\text { Massa } \\
\text { placa }[\mathrm{g}]\end{array}$ \\
\hline 1 & Aço A36 & 3,2 & $250 \times 100$ & 592,8 \\
\hline 2 & Aço A36 & 6,35 & $250 \times 100$ & 1263,1 \\
\hline 3 & Aço A36 & 9,5 & $250 \times 100$ & 1843,1 \\
\hline
\end{tabular}

Como pode ser observado nas Tab. 2 a massa de $\mathrm{N}_{2} \mathrm{~L}$ evaporado devido a trocar de calor entre a placa teste e o $\mathrm{N}_{2} \mathrm{~L}$ (calculada através da diferença de massa apresentada na Eq. (4)) apresenta bons resultados com uma diferença não maior a 1,5 $\%$ para as placas de $3,2 \mathrm{~mm}, 0,5 \%$ para as placas de $6,35 \mathrm{~mm}$ e $0,64 \%$ para as placas de $9,5 \mathrm{~mm}$. Também observar-se que o tempo de estabilização (diferença de tempo desde que entra a placa teste até alcançar o ponto de equilíbrio - $\mathrm{t}_{\mathrm{est}}$ ) foi constante para o mesmo tipo de chapa e aumento de acordo com a espessura da placa teste. A Figura 4 apresenta os sinais do processo de evaporação para as placas com diferentes espessuras utilizadas, onde se pode observar boa repetibilidade do calorímetro de $\mathrm{N}_{2} \mathrm{~L}$ proposto.

Tabela 2. Resultados da massa evaporada de $\mathrm{N}_{2} \mathrm{~L}$ com placas em condições ambientais

\begin{tabular}{|c|c|c|c|c|c|}
\hline Placa teste & $\mathrm{mi} \mathrm{N}_{2} \mathrm{~L}[\mathrm{~g}]$ & $\mathrm{mf} \mathrm{N}_{2} \mathrm{~L}[\mathrm{~g}]$ & massa placa $[\mathrm{g}]$ & $\mathrm{N}_{2}$ evap. $[\mathrm{g}]$ & $\mathrm{t}_{\text {est }}[\mathrm{s}]$ \\
\hline Placa 3,2 $\mathrm{mm}$ & 9190,3 & 9531,7 & 579,5 & 238,1 & 61,0 \\
\hline Réplica 1 & 9190,5 & 9530,2 & 579,5 & 239,8 & 61,0 \\
\hline Réplica 2 & 9190,4 & 9525,5 & 579,5 & 244,4 & 61,0 \\
\hline \multicolumn{7}{|c|}{ Média } & $240,8 \pm 3,3$ & \\
\hline Placa 6,35 mm & 9558,2 & 10318,4 & 1261,6 & 501,4 & 109,0 \\
\hline Réplica 1 & 9558 & 10313,2 & 1261,6 & 506,4 & 109,0 \\
\hline Réplica 2 & 9558,1 & 10315,1 & 1261,6 & 504,6 & 109,0 \\
\hline \multicolumn{7}{|c|}{} & 10770,7 & 1843,1 & 732,0 & 157,0 \\
\hline Placa 9,5 mm & 9659,6 & 10772,4 & 1843,1 & 730,3 & 157,0 \\
\hline Réplica 1 & 9659,6 & 10763,2 & 1843,1 & 739,1 & 157,0 \\
\hline Réplica 2 & 9659,2 & & Média & $733,8 \pm 4,7$ & \\
\hline \multicolumn{7}{|c|}{} \\
\hline
\end{tabular}



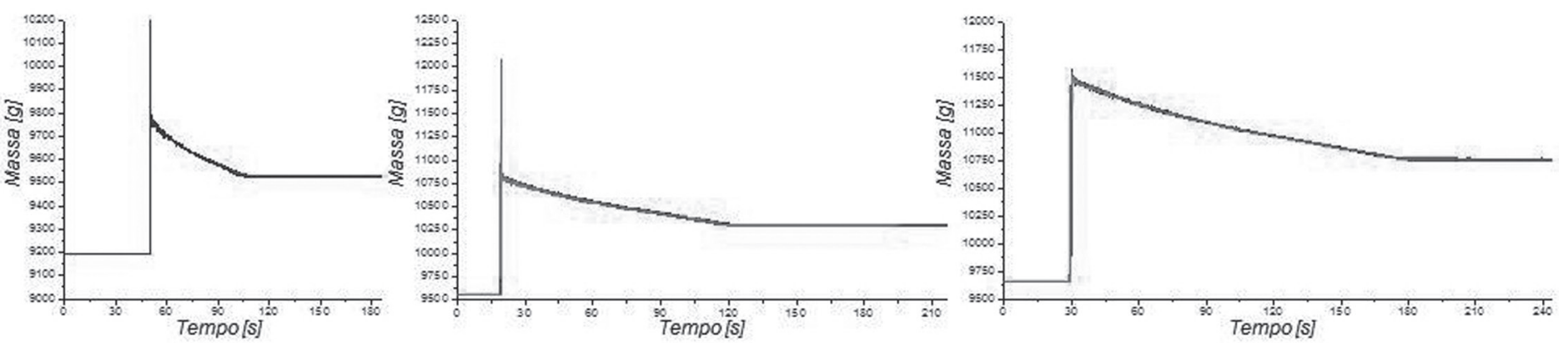

Figura 4. Comportamento da massa evaporada de $\mathrm{N}_{2} \mathrm{~L}$ para placas de teste de diferentes espessuras $(3,2 ; 6,35$ e 9,5 mm da esquerda para direita)

\section{Medição do aporte térmico e rendimento térmico}

Os processos investigados foram GTAW (TIG), GMAW (MIG/MAG) convencional utilizando transferências metálicas por curto-circuito e goticular (spray) e processos derivativos GMAW. A movimentação da tocha de soldagem foi realizada através de um movimentador linear que garante um movimento constante e sem variação do comprimento do arco. A sujeição da placa teste foi realizada através do sistema de garras apresentado no Item 3, que tem como segunda função deslocar a placa teste ate o sistema de medição de massa evaporada de $\mathrm{N}_{2} \mathrm{~L}$. O sistema de aquisição dos sinais elétricos de tensão e corrente da fonte de soldagem foi realizada através de uma placa desenvolvida no Laprosolda que adéqua os sinais de corrente e tensão para ser processados pela placa de aquisição NI USB 6215 com taxa de aquisição de dados de $2000 \mathrm{~Hz}$.

$\mathrm{O}$ cálculo da energia de soldagem (energia de soldagem por comprimento de cordão) é realizado através de Eq. 5, onde a potência instantânea, Eq. 6, é definida como o produto da tensão e corrente ponto a ponto, representando de forma mais precisa o comportamento da energia levando em consideração qualquer variação na tensão e corrente durante o processo de soldagem [12]. Ressaltando-se que este cálculo é realizado unicamente durante o tempo de arco aberto (processo de formação do cordão).

$\mathrm{E}_{\text {sold }}=\mathrm{P}_{\text {inst }} / \mathrm{Vs},[\mathrm{J} / \mathrm{mm}]$

Onde, $\mathrm{P}_{\text {inst }}$ é a potência instantânea [W]; Vs é a velocidade de soldagem $[\mathrm{mm} / \mathrm{s}]$.

$\mathrm{P}_{\text {inst }}=\left(\sum_{\mathrm{i}=1}^{\mathrm{n}}\left(\mathrm{U}_{\mathrm{i}} * \mathrm{I}_{\mathrm{i}}\right)\right) / \mathrm{n}$

Onde, $\mathrm{U}_{\mathrm{i}}$ é a tensão do processo de soldagem [V]; $\mathrm{I}_{\mathrm{i}}$ é a corrente do processo de soldagem [A]; n é numero de dados durante o período de arco aberto.

Para o cálculo do aporte térmico foi seguido o procedimento proposto no Item 2, onde se devem levar em consideração as duas etapas de cálculo (placa com energia imposta do processo de soldagem e placas em condições ambientais depois do processo de soldagem). O rendimento térmico do processo é definido como a relação entre o aporte térmico $\left(E_{\text {imp }}\right)$ e a energia de soldagem $\left(\mathrm{E}_{\text {sold }}\right)$, como é apresentado na Eq. 7. Assim, devese destacar esta equação, pois o pessoal técnico de soldagem ainda utiliza indiscriminadamente esta nomenclatura, sem fazer distinção entre energia de soldagem (mais facilmente medida durante o processo) e aporte térmico (a ser medido via calorimetria).

$\eta_{\text {term }}=\frac{E_{\text {imp }}}{E_{\text {sold }}} * 100 \%$

\subsection{Processo GTAW}

$\mathrm{Na}$ soldagem GTAW, as soldas foram realizadas na polaridade negativa, eletrodo EW-Th2\% com diâmetro de 2,4 mm e ângulo de ponta de $60^{\circ}$, proteção gasosa de argônio $100 \%$ e vazão de $15 \mathrm{~L} / \mathrm{min}$. As soldas foram realizadas sobre placa ASTM A36 na posição plana com dimensões de 200 x 100 x $3,2 \mathrm{~mm}$. Foi proposta a variação da corrente em dois níveis $(100$ e $150 \mathrm{~A})$, dois comprimento de arco (3 e $5 \mathrm{~mm})$, três níveis de velocidade de soldagem $(15,20$ e $25 \mathrm{~cm} / \mathrm{min}$.) e três níveis comprimentos de cordão de $\operatorname{solda}(8,12$ e $16 \mathrm{~cm})$ com o objetivo de verificar a influência de cada um destes parâmetros sobre o rendimento térmico do processo. Neste caso foi realizado um planejamento fatorial completo com 36 testes no total (Tab. 3).

A Tabela 4 apresenta os resultados obtidos para a variação dos parâmetros propostos no planejamento experimental. Os resultados foram analisados estatisticamente com análise de variância para cada um dos parâmetros de soldagem propostos. Deve-se ressaltar que cada valor representa uma média de duas medições.

O comportamento do rendimento térmico tem uma tendência significativa a diminuir com o aumento da corrente, apresentando uma queda de $71 \%$ a $69,7 \%$ para $100 \mathrm{~A}$ e 150 A respectivamente como é apresentado na Fig. 5 (a). Este fato é devido, provavelmente, às perdas de calor por convecção e radiação, que têm uma influência maior que o aumento da corrente. Niles [2] em um estudo realizado para TIG variando diferentes parâmetros, como a corrente e os gases de proteção, reporta que o rendimento térmico cai de $60 \%$ a $35 \%$ para correntes de 125 A e $225 \mathrm{~A}$, respectivamente. A variação de corrente no processo TIG, mantendo-se constantes os outros parâmetros de soldagem $\left(\mathrm{L}_{\mathrm{a}}\right.$ e $\left.\mathrm{V}_{\mathrm{s}}\right)$, apresenta variação na penetração para o cordão de solda como é conhecido [5], mas objetivo deste trabalho não é 
Tabela 3. Planejamento experimental para a avaliação do rendimento térmico no processo TIG

\begin{tabular}{|c|c|c|c|c|c|c|c|c|c|c|c|c|c|c|c|c|c|c|}
\hline $\mathrm{I}[\mathrm{A}]$ & \multicolumn{18}{|c|}{100} \\
\hline $\mathrm{L}_{\mathrm{a}}[\mathrm{mm}]$ & \multicolumn{9}{|c|}{3} & \multicolumn{9}{|c|}{5} \\
\hline $\mathrm{V}_{\mathrm{s}}[\mathrm{cm} / \mathrm{min}]$ & \multicolumn{3}{|c|}{15} & \multicolumn{3}{|c|}{20} & \multicolumn{3}{|c|}{25} & \multicolumn{3}{|c|}{15} & \multicolumn{3}{|c|}{20} & \multicolumn{3}{|c|}{25} \\
\hline $\mathrm{L}_{\mathrm{c}}[\mathrm{cm}]$ & 8 & 12 & 16 & 8 & 12 & 16 & 8 & 12 & 16 & 8 & 12 & 16 & 8 & 12 & 16 & 8 & 12 & 16 \\
\hline
\end{tabular}

\begin{tabular}{|c|c|c|c|c|c|c|c|c|c|c|c|c|c|c|c|c|c|c|}
\hline $\mathrm{I}[\mathrm{A}]$ & \multicolumn{18}{|c|}{150} \\
\hline $\mathrm{L}_{\mathrm{a}}[\mathrm{mm}]$ & \multicolumn{9}{|c|}{3} & \multicolumn{9}{|c|}{5} \\
\hline $\mathrm{V}_{\mathrm{s}}[\mathrm{cm} / \mathrm{min}]$ & \multicolumn{3}{|c|}{15} & \multicolumn{3}{|c|}{20} & \multicolumn{3}{|c|}{25} & \multicolumn{3}{|c|}{15} & \multicolumn{3}{|c|}{20} & \multicolumn{3}{|c|}{25} \\
\hline $\mathrm{L}_{\mathrm{c}}[\mathrm{cm}]$ & 8 & 12 & 16 & 8 & 12 & 16 & 8 & 12 & 16 & 8 & 12 & 16 & 8 & 12 & 16 & 8 & 12 & 16 \\
\hline
\end{tabular}

I representa corrente de soldagem, $L_{a}$ representa comprimento do arco, $V_{s}$ representa velocidade de soldagem e $L_{c}$ representa comprimento do cordão de solda.

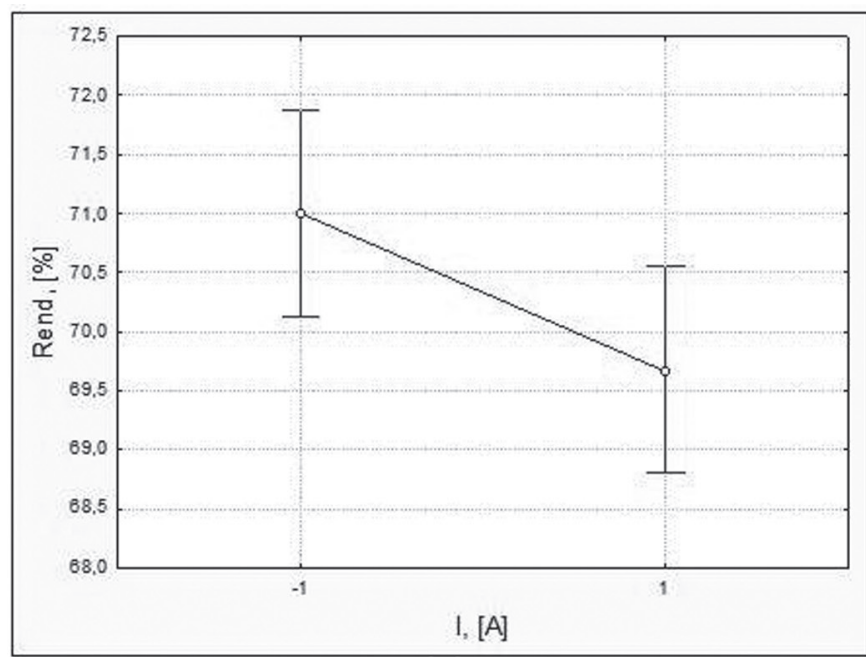

(a)

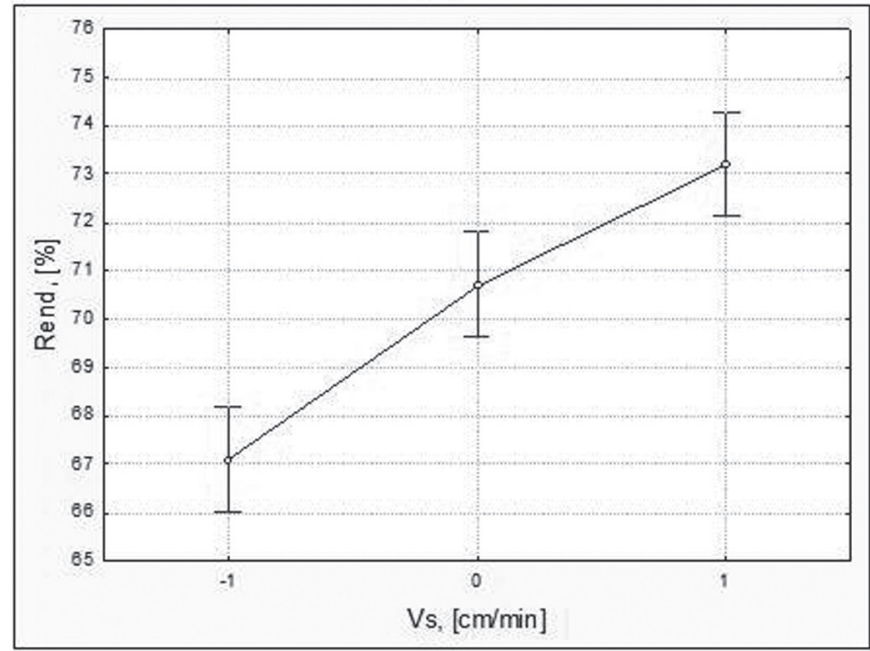

(c)

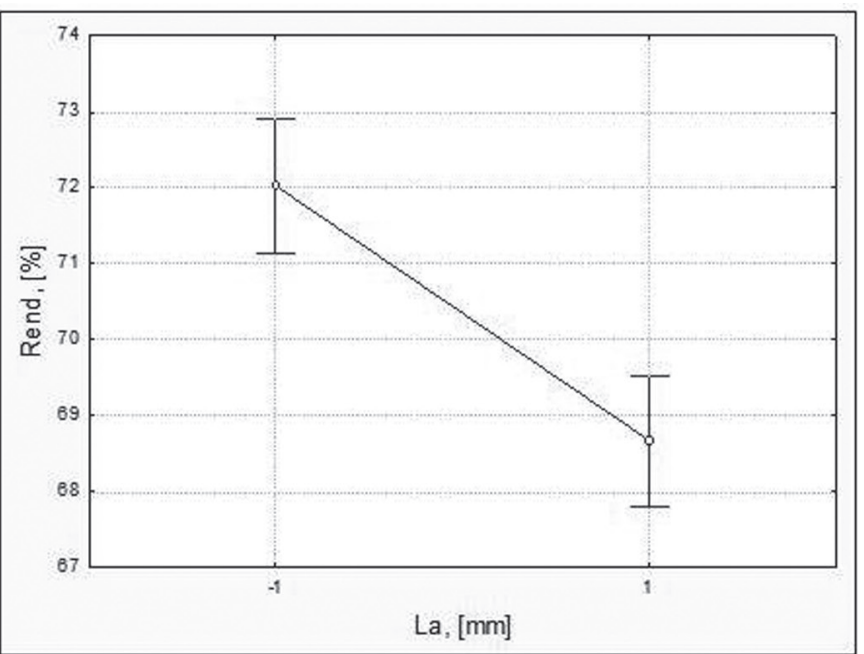

(b)

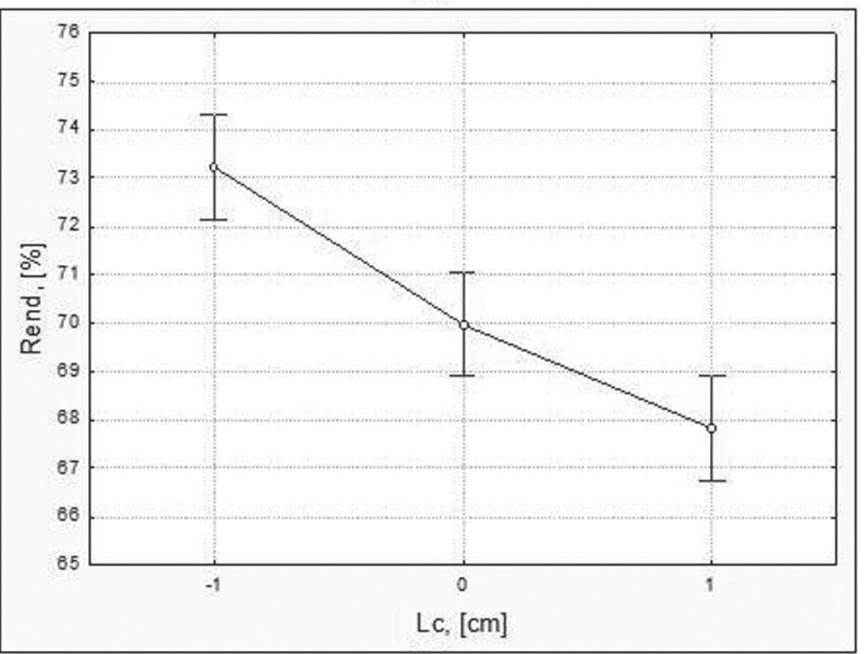

(d)

Figura 5. (a) Efeito da corrente (I) sob a rendimento térmico, (b) efeito do comprimento do arco ( $\mathrm{L}_{\mathrm{a}}$ ) sob a rendimento térmico, (c) efeito da velocidade de soldagem $\left(\mathrm{V}_{\mathrm{s}}\right)$ sob o rendimento termico e $(\mathrm{d})$ efeito do comprimento do cordão $\left(\mathrm{L}_{\mathrm{c}}\right)$ sob o rendimento térmico 
Tabela 4. Resultados para o planejamento experimental proposto para o processo TIG

\begin{tabular}{|c|c|c|c|c|c|c|c|c|c|}
\hline $\mathrm{I}[\mathrm{A}]$ & $\mathrm{L}_{\mathrm{a}}[\mathrm{mm}]$ & $\mathrm{V}_{\mathrm{s}}[\mathrm{cm} / \mathrm{min}]$ & $\mathrm{L}_{\mathrm{c}}[\mathrm{cm}]$ & $\mathrm{U}_{\mathrm{m}}[\mathrm{V}]$ & $\mathrm{I}_{\mathrm{m}}[\mathrm{A}]$ & $\mathrm{P}_{\text {inst }}[\mathrm{W}]$ & $\mathrm{E}_{\text {sold }}[\mathrm{J} / \mathrm{mm}]$ & $\mathrm{E}_{\mathrm{imp}}[\mathrm{J} / \mathrm{mm}]$ & $\eta_{\text {term }}[\%]$ \\
\hline \multirow{18}{*}{100} & \multirow{9}{*}{3} & \multirow{3}{*}{15} & 8 & 10,9 & 102 & 1112 & 445 & 319 & 71,8 \\
\hline & & & 12 & 10,6 & 101 & 1069 & 428 & 273 & 63,7 \\
\hline & & & 16 & 10,2 & 101 & 1029 & 412 & 287 & 69,6 \\
\hline & & \multirow{3}{*}{20} & 8 & 10,8 & 102 & 1094 & 328 & 259 & 78,9 \\
\hline & & & 12 & 10,1 & 102 & 1028 & 309 & 229 & 74,3 \\
\hline & & & 16 & 10,3 & 102 & 1050 & 315 & 225 & 71,4 \\
\hline & & \multirow{3}{*}{25} & 8 & 10,7 & 102 & 1087 & 261 & 205 & 78,7 \\
\hline & & & 12 & 10,4 & 102 & 1063 & 255 & 194 & 76,0 \\
\hline & & & 16 & 9,9 & 102 & 1011 & 243 & 167 & 69,0 \\
\hline & \multirow{9}{*}{5} & \multirow{3}{*}{15} & 8 & 11,3 & 101 & 1144 & 458 & 314 & 68,6 \\
\hline & & & 12 & 11,2 & 101 & 1130 & 452 & 302 & 66,9 \\
\hline & & & 16 & 10,9 & 101 & 1103 & 441 & 301 & 68,3 \\
\hline & & \multirow{3}{*}{20} & 8 & 11,3 & 101 & 1139 & 342 & 246 & 72,0 \\
\hline & & & 12 & 11,1 & 101 & 1119 & 336 & 228 & 67,9 \\
\hline & & & 16 & 11,1 & 101 & 1121 & 336 & 222 & 65,9 \\
\hline & & \multirow{3}{*}{25} & 8 & 12,0 & 101 & 1215 & 292 & 216 & 74,2 \\
\hline & & & 12 & 11,6 & 101 & 1174 & 282 & 203 & 72,0 \\
\hline & & & 16 & 11,0 & 101 & 1110 & 266 & 184 & 69,1 \\
\hline \multirow{18}{*}{150} & \multirow{9}{*}{3} & \multirow{3}{*}{15} & 8 & 11,1 & 150 & 1665 & 666 & 474 & 71,2 \\
\hline & & & 12 & 10,6 & 150 & 1594 & 638 & 426 & 66,7 \\
\hline & & & 16 & 10,5 & 150 & 1580 & 632 & 411 & 65,0 \\
\hline & & \multirow{3}{*}{20} & 8 & 10,7 & 150 & 1595 & 478 & 355 & 74,2 \\
\hline & & & 12 & 10,1 & 150 & 1517 & 455 & 334 & 73,3 \\
\hline & & & 16 & 10,8 & 150 & 1622 & 487 & 334 & 68,6 \\
\hline & & \multirow{3}{*}{25} & 8 & 10,7 & 150 & 1595 & 383 & 299 & 78,1 \\
\hline & & & 12 & 10,0 & 150 & 1500 & 360 & 268 & 74,4 \\
\hline & & & 16 & 10,2 & 150 & 1534 & 368 & 263 & 71,5 \\
\hline & \multirow{9}{*}{5} & \multirow{3}{*}{15} & 8 & 12,6 & 151 & 1891 & 756 & 513 & 67,9 \\
\hline & & & 12 & 11,6 & 150 & 1744 & 698 & 451 & 64,6 \\
\hline & & & 16 & 11,9 & 150 & 1785 & 714 & 435 & 60,9 \\
\hline & & \multirow{3}{*}{20} & 8 & 12,1 & 150 & 1825 & 547 & 383 & 69,9 \\
\hline & & & 12 & 11,4 & 150 & 1715 & 514 & 345 & 67,0 \\
\hline & & & 16 & 11,4 & 150 & 1708 & 512 & 334 & 65,3 \\
\hline & & \multirow{3}{*}{25} & 8 & 11,7 & 150 & 1755 & 421 & 309 & 73,3 \\
\hline & & & 12 & 11,6 & 150 & 1742 & 418 & 305 & 72,9 \\
\hline & & & 16 & 11,7 & 150 & 1756 & 421 & 292 & 69,3 \\
\hline
\end{tabular}


estudar as características geométricas do cordão para diferentes condições de soldagem, mas pode dar outra noção do porquê se apresenta a queda de rendimento térmico com o aumento da corrente. A Figura 6 mostra o esquema do formato de cordão obtido para os dois níveis de corrente (100 A e 150 A), observando-se que o cordão de $150 \mathrm{~A}$ tem uma maior penetração e reforço de raiz, comparados com o cordão de $100 \mathrm{~A}$. Com o aumento da área do reforço da raiz, aumenta-se a troca de calor com o meio ambiente por convecção e radiação como pode ser verificado na Fig. 7.

Assim, acredita-se que uma menor espessura de placa teste apresentaria menores rendimentos térmicos, assim como foi citado por Zijp [9] onde o rendimento térmico foi menor para chapas com espessura de $2 \mathrm{~mm}$ quando comparadas com chapas de 5 e $10 \mathrm{~mm}$ de espessura. Esta comparação do rendimento térmico para diferentes espessuras de corpo de prova foram realizadas com corrente constante de $100 \mathrm{~A}$ e variação do comprimento do arco.

Apesar de que o incremento do arco (tensão do arco) aumenta o aporte térmico, o rendimento térmico apresenta uma queda significativa, como pode ser observado na Fig. 5 (b). Este efeito pode ser atribuído a maiores perdas de calor na coluna do arco, onde um maior comprimento do arco leva a uma maior área de contado com o ambiente, como é apresentado esquematicamente na Fig. 8.

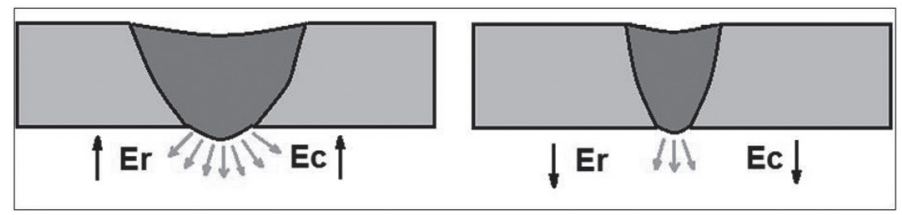

Figura 6. Esquema da forma do cordão para processo TIG CC- com corrente de $150 \mathrm{~A}$ e $100 \mathrm{~A}$ em placa com $3 \mathrm{~mm}$ de espessura

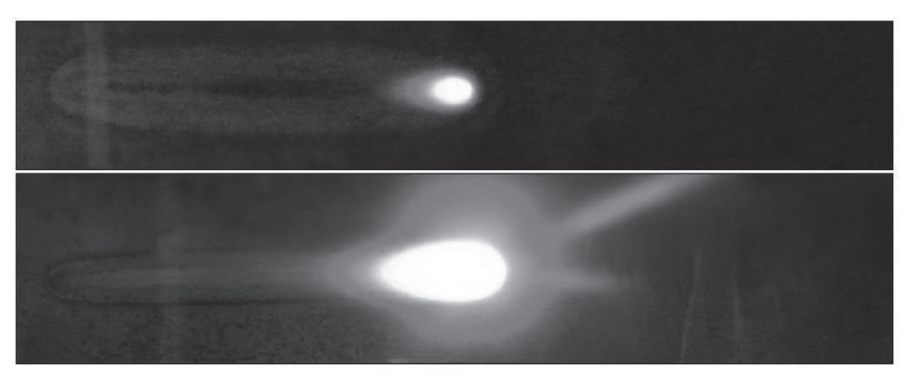

Figura 7. Raiz do cordão durante o processo de soldagem TIG CC- com parâmetros de soldagem: $\mathrm{L}_{\mathrm{a}}=3 \mathrm{~mm}, \mathrm{~V}_{\mathrm{s}}=20 \mathrm{~cm} / \mathrm{min}$., proteção gasosa argônio puroe (a) $100 \mathrm{~A} \mathrm{e} \mathrm{(b)} 150 \mathrm{~A}$

A eficiência térmica apresenta um aumento significativo com o aumento da velocidade de soldagem, como mostrado na Fig. 5 (c). Sugere-se que isto é em função da alteração de regime de transferência de calor. Uma vez que quanto menor a velocidade de soldagem, maior será penetração e maiores perdas térmicas acontecerão do lado da raiz da solda. Além disto, há de se considerar que em um cordão feito mais rapidamente, há menor tempo de se trocar calor entre a chapa e o meio ambiente, até o momento em que a placa de teste entra no $\mathrm{N}_{2} \mathrm{~L}$. Vale ressaltar ainda que esta consideração de aumentar o rendimento térmico com o aumento da velocidade de soldagem vai até certo ponto, já que velocidades de soldagem demasiada elevadas também podem afetar de forma negativa o calor imposto $[10,11]$.

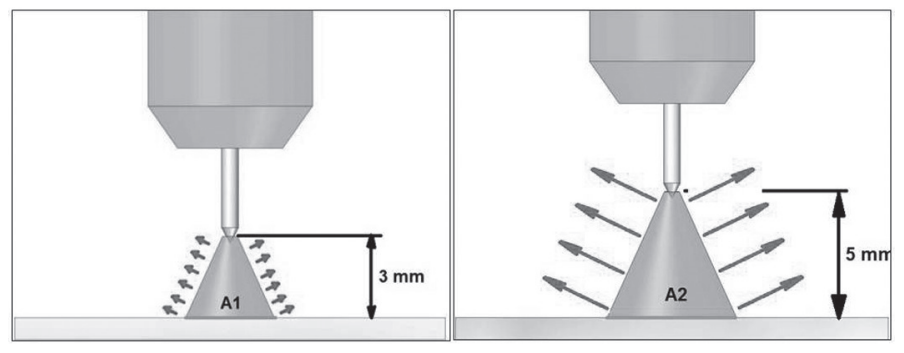

Figura 8. Variação do comprimento do arco em processo TIG

Por fim, observa-se na Fig. 5 (d) que o rendimento térmico sofre uma queda significativa para maiores comprimentos de cordão, onde o tempo de formação do cordão afeta diretamente o calor imposto, já que as perdas de calor durante a formação do mesmo influenciam de forma negativa a eficiência térmica do processo. Isto reflete uma característica importante do ensaio proposto, que é o tempo entre a formação do cordão e o "mergulho" da placa de teste no nitrogênio líquido. Este tempo implica em trocas térmicas com o meio ambiente, sendo uma limitação do ensaio. Entretanto, pela automação executada, garante-se uma grande repetibilidade em todas as medições.

O rendimento térmico para o processo TIG CC- foi de $70 \%$ e um rendimento máximo de $79 \%$ e mínimo de $61 \%$, para as condições de soldagem preestabelecidas para este trabalho. Sendo estes valores muito próximos comparados com trabalhos já realizados por outros autores $[9,12,13,14]$, que reportando rendimento térmico de $67 \%$ a $80 \%$.

\subsection{Processos GMAW convencionais}

Após as soldagens GTAW, os planejamentos experimentais mostrados nas Tab. 5 e 6 apresentam os ensaios propostos para a execução das soldagens GMAW convencional (fonte em modo tensão constante) utilizando-se respectivamente transferência metálica por curto-circuito e goticular (spray). Para a transferência por curto-circuito utilizou-se uma distância bico de contato peça (DBCP) de $12 \mathrm{~mm}$ e para goticular de 21 $\mathrm{mm}$.

Destaca-se que a ideia destes planejamentos é prover uma avaliação geral das grandezas térmicas passíveis de serem medidas (aporte térmico e rendimento térmico). Assim, optou-se por efetuar um planejamento experimental mais simples. Neste caso, ao se alterar a velocidade de alimentação, sem se alterar a tensão de referência, o comprimento do arco irá se alterar, o que possivelmente trará alterações nas grandezas térmicas mencionadas. Além disto, a taxa de deposição se alterou ao se alterar a relação entre a velocidade de alimentação e de soldagem. 
Tabela 5. Planejamento experimental proposto para a soldagem GMAW curto-circuito convencional

\begin{tabular}{|c|c|c|c|c|c|}
\hline Teste & $\begin{array}{c}\mathrm{DBCP} \\
{[\mathrm{mm}]}\end{array}$ & $\mathrm{U}[\mathrm{V}]$ & $\mathrm{V}_{\mathrm{a}}[\mathrm{m} / \mathrm{min}]$ & $\mathrm{V}_{\mathrm{s}}[\mathrm{cm} / \mathrm{min}]$ & $\begin{array}{c}\mathrm{L}_{\mathrm{c}} \\
{[\mathrm{cm}]}\end{array}$ \\
\hline 1 & \multirow{8}{*}{12} & \multirow{8}{*}{19,0} & \multirow{4}{*}{3,0} & \multirow{2}{*}{30} & 10 \\
\hline 2 & & & & & 15 \\
\hline 3 & & & & \multirow{2}{*}{40} & 10 \\
\hline 4 & & & & & 15 \\
\hline 5 & & & \multirow{4}{*}{4,0} & \multirow{2}{*}{30} & 10 \\
\hline 6 & & & & & 15 \\
\hline 7 & & & & \multirow{2}{*}{40} & 10 \\
\hline 8 & & & & & 15 \\
\hline
\end{tabular}

Tabela 6.Planejamento experimental proposto para a soldagem GMAW goticular

\begin{tabular}{|c|c|c|c|c|c|}
\hline Teste & $\begin{array}{c}\text { DBCP } \\
{[\mathrm{mm}]}\end{array}$ & $\begin{array}{c}\mathrm{U} \\
{[\mathrm{V}]}\end{array}$ & $\mathrm{V}_{\mathrm{a}}[\mathrm{m} / \mathrm{min}]$ & $\mathrm{V}_{\mathrm{s}}[\mathrm{cm} / \mathrm{min}]$ & $\begin{array}{c}\mathrm{L}_{\mathrm{c}} \\
{[\mathrm{cm}]}\end{array}$ \\
\hline 1 & \multirow{8}{*}{21} & \multirow{8}{*}{29,0} & \multirow{4}{*}{6,5} & \multirow{2}{*}{30} & 10 \\
\hline 2 & & & & & 15 \\
\hline 3 & & & & \multirow{2}{*}{40} & 10 \\
\hline 4 & & & & & 15 \\
\hline 5 & & & \multirow{4}{*}{8,5} & \multirow{2}{*}{30} & 10 \\
\hline 6 & & & & & 15 \\
\hline 7 & & & & \multirow{2}{*}{40} & 10 \\
\hline 8 & & & & & 15 \\
\hline
\end{tabular}

Tabela 7. Valores obtidos pelo calorímetro de N2L para processo GMAW curto-circuito convencional

\begin{tabular}{|c|c|c|c|c|c|c|c|c|c|}
\hline $\mathrm{U}[\mathrm{V}]$ & $\mathrm{V}_{\mathrm{a}}[\mathrm{m} / \mathrm{min}]$ & $\mathrm{V}_{\mathrm{s}}[\mathrm{cm} / \mathrm{min}]$ & $\mathrm{L}_{\mathrm{c}}[\mathrm{cm}]$ & $\mathrm{U}_{\mathrm{m}}[\mathrm{V}]$ & $\mathrm{I}_{\mathrm{m}}[\mathrm{A}]$ & $\mathrm{P}_{\text {inst }}[\mathrm{W}]$ & $\mathrm{E}_{\text {sold }}[\mathrm{J} / \mathrm{mm}]$ & $\mathrm{E}_{\mathrm{imp}}[\mathrm{J} / \mathrm{mm}]$ & $\boldsymbol{\eta}_{\text {term }}[\%]$ \\
\hline \multirow{8}{*}{19,0} & \multirow{4}{*}{3,0} & \multirow{2}{*}{30} & 10 & 19,5 & 122 & 2480 & 496 & 407 & 82,1 \\
\hline & & & 15 & 19,6 & 126 & 2562 & 512 & 398 & 77,7 \\
\hline & & \multirow{2}{*}{40} & 10 & 19,5 & 123 & 2495 & 374 & 286 & 76,3 \\
\hline & & & 15 & 19,5 & 128 & 2593 & 389 & 303 & 77,9 \\
\hline & \multirow{4}{*}{4,0} & \multirow{2}{*}{30} & 10 & 20,0 & 164 & 3341 & 668 & 516 & 77,2 \\
\hline & & & 15 & 20,0 & 166 & 3363 & 673 & 525 & 78,0 \\
\hline & & \multirow{2}{*}{40} & 10 & 20,1 & 167 & 3399 & 510 & 411 & 80,7 \\
\hline & & & 15 & 19,9 & 168 & 3384 & 508 & 391 & 77,1 \\
\hline
\end{tabular}

Tabela 8. Valores obtidos pelo calorímetro de N2L para processo GMAW transferência goticular

\begin{tabular}{|c|c|c|c|c|c|c|c|c|c|}
\hline $\mathrm{U}[\mathrm{V}]$ & $\mathrm{V}_{\mathrm{a}}[\mathrm{m} / \mathrm{min}]$ & $\mathrm{V}_{\mathrm{s}}[\mathrm{cm} / \mathrm{min}]$ & $\mathrm{L}_{\mathrm{c}}[\mathrm{cm}]$ & $\mathrm{U}_{\mathrm{m}}[\mathrm{V}]$ & $\mathrm{I}_{\mathrm{m}}[\mathrm{A}]$ & $\mathrm{P}_{\text {inst }}[\mathrm{W}]$ & $\mathrm{E}_{\text {sold }}[\mathrm{J} / \mathrm{mm}]$ & $\mathrm{E}_{\mathrm{imp}}[\mathrm{J} / \mathrm{mm}]$ & $\boldsymbol{\eta}_{\text {term }}[\%]$ \\
\hline \multirow{8}{*}{29,0} & \multirow{4}{*}{6,5} & \multirow{2}{*}{30} & 10 & 28,5 & 247 & 7039 & 1408 & 931 & 66,2 \\
\hline & & & 15 & 28,6 & 248 & 7078 & 1416 & 991 & 70,0 \\
\hline & & \multirow{2}{*}{40} & 10 & 28,6 & 246 & 7019 & 1053 & 775 & 73,6 \\
\hline & & & 15 & 28,8 & 244 & 6985 & 1048 & 750 & 71,5 \\
\hline & \multirow{4}{*}{8,5} & \multirow{2}{*}{30} & 10 & 28,0 & 282 & 7909 & 1582 & 1241 & 78,5 \\
\hline & & & 15 & 27,6 & 286 & 7985 & 1597 & 1258 & 78,8 \\
\hline & & \multirow{2}{*}{40} & 10 & 28,0 & 281 & 7881 & 1182 & 900 & 76,1 \\
\hline & & & 15 & 28,1 & 287 & 8055 & 1208 & 897 & 74,2 \\
\hline
\end{tabular}

Os resultados destes dois planejamentos experimentais estão apresentados nas Tabs. 7 e 8 . A primeira observação é que a transferência metálica goticular, em função dos parâmetros utilizados, atingiu em média o dobro da potência instantânea (7494 W) se comparada com a transferência por curto-circuito (2952 W). Este resultado é uma indicação da maior tensão e maior corrente utilizadas na transferência goticular. Com relação à eficiência térmica, a transferência por curto-circuito apresentou valores maiores em média $(79,4 \%)$ se comparada à transferência goticular $(73,6$ $\%$ ). Este é um indicativo de que menores arcos de soldagem e menores níveis de corrente determinam menores perdas térmicas e, consequentemente, maiores eficiências térmicas. Em comparação com a literatura valores para rendimento térmico da ordem de $86 \%$ para a transferência por curto-circuito e $73 \%$ para a transferência goticular são apresentados [15].

\subsection{Processos GMAW derivativos}

Devido à importância crescente dos processos GMAW com transferência controlada (processos derivativos), são propostos nas Tab. 9 a 11 ensaios para avaliar tais processos no tocante a suas eficiências térmicas. Estes processos utilizam diferentes tecnologias: STT (Surface Tension Transfer), RMD (Regulated Metal Deposition), CMT (Cold Metal Transfer), GMAW-P (Pulsado) e GMAW-PV (Polaridade Variável). Neste trabalho, para maior facilidade de comunicação escrita, a nomenclatura "Processo GMAW com transferência metálica por curto-circuito controlado utilizando-se tecnologia STT/RMD/CMT" será resumida na forma "Processo STT/RMD/CMT". Destaca-se que a primeira nomenclatura é aquela tecnicamente correta, mas para simplificação escrita, a segunda será adotada. 
Tabela 9. Planejamento experimental para o calorímetro de N2L para os processos GMAW derivativos STT, RMD e CMT para os dois níveis de corrente: 115 A e 155 A

\begin{tabular}{|c|c|c|c|c|c|}
\hline Processo & $\mathrm{V}_{\mathrm{s}}[\mathrm{cm} / \mathrm{min}]$ & $\mathrm{V}_{\mathrm{a}}[\mathrm{m} / \mathrm{min}]$ & $\mathrm{DBCP}[\mathrm{mm}]$ & Parâmetro específico & $\mathrm{I}_{\mathrm{m}}[\mathrm{A}]$ \\
\hline \multirow{4}{*}{ STT } & 12 & 2,3 & 12 & $\mathrm{Ip}=300 \mathrm{~A} ; \mathrm{Ib}=80$ A; Tailout=5 & 118 \\
\cline { 2 - 6 } & 12 & 2,3 & 12 & $\mathrm{Ip}=300 \mathrm{~A} ; \mathrm{Ib}=80$ A; Tailout=5 & 119 \\
\cline { 2 - 6 } & 18 & 3,3 & 12 & $\mathrm{Ip}=300 \mathrm{~A} ; \mathrm{Ib}=80$ A; Tailout=5 & 155 \\
\cline { 2 - 6 } & 18 & 3,3 & 12 & $\mathrm{Ip}=300 \mathrm{~A} ; \mathrm{Ib}=80$ A; Tailout=5 & 154 \\
\hline \multirow{5}{*}{ RMD } & 12 & 2,3 & 18 & Trim=80; ArcControl=25 & 118 \\
\cline { 2 - 6 } & 12 & 2,3 & 18 & Trim=80; ArcControl=25 & 117 \\
\cline { 2 - 6 } & 18 & 3,3 & 16 & Trim=80; ArcControl=25 & 154 \\
\hline \multirow{5}{*}{ CMT } & 18 & 3,3 & 16 & Trim=80; ArcControl=25 & 119 \\
\cline { 2 - 6 } & 12 & 2,3 & 12 & Arclength= -20 & 118 \\
\cline { 2 - 6 } & 18 & 2,3 & 12 & Arclength= -20 & 157 \\
\hline
\end{tabular}

Tabela 10. Planejamento experimental para o calorímetro de N2L para o processo derivativo GMAW-P (Pulsado) para os dois níveis de corrente utilizados: 115 A e $155 \mathrm{~A}$

\begin{tabular}{|c|c|c|c|c|c|c|}
\hline $\mathrm{I}_{\mathrm{p}}[\mathrm{A}]$ & $\mathrm{t}_{\mathrm{p}}[\mathrm{ms}]$ & $\mathrm{I}_{\mathrm{b}}[\mathrm{A}]$ & $\mathrm{t}_{\mathrm{b}}[\mathrm{ms}]$ & $\mathrm{DBCP}[\mathrm{mm}]$ & $\mathrm{V}_{\mathrm{a}}[\mathrm{m} / \mathrm{min}]$ & $\mathrm{I}_{\mathrm{m}}[\mathrm{A}]$ \\
\hline 275 & 7,6 & 60 & 24 & 22 & 3,0 & 115 \\
\hline 275 & 5,5 & 60 & 7,0 & 22 & 4,0 & 156 \\
\hline
\end{tabular}

Ip é a corrente de pico; tp é o tempo de pico; $I b$ é a corrente de base e $t b$ é o tempo de base

Tabela 11. Planejamento experimental para o calorímetro de N2L para o processo derivativo GMAW-PV (Polaridade Variável) para os dois níveis de corrente utilizados: $115 \mathrm{~A}$ e $155 \mathrm{~A}$

\begin{tabular}{|c|c|c|c|c|c|c|c|c|c|}
\hline $\mathrm{I}_{\mathrm{p}}[\mathrm{A}]$ & $\mathrm{I}_{\mathrm{b}}[\mathrm{A}]$ & $\mathrm{I}_{\mathrm{n}}[\mathrm{A}]$ & $\mathrm{t}_{\mathrm{p}}[\mathrm{ms}]$ & $\mathrm{t}_{\mathrm{b} 1}[\mathrm{~ms}]$ & $\mathrm{t}_{\mathrm{b} 2}[\mathrm{~ms}]$ & $\mathrm{t}_{\mathrm{n}}[\mathrm{ms}]$ & $\mathrm{V}_{\mathrm{a}}[\mathrm{m} / \mathrm{min}]$ & $\% \mathrm{EN}$ & $\mathrm{I}_{\mathrm{m}}[\mathrm{A}]$ \\
\hline 250 & 40 & -85 & 6,9 & 8,75 & 2,0 & 6,0 & 3,1 & $20 \%$ & 114 \\
\hline 250 & 40 & -85 & 6,9 & 9,75 & 1,0 & 5,5 & 3,1 & $20 \%$ & 114 \\
\hline 250 & 40 & -125 & 5,85 & 2,5 & 1,44 & 3,5 & 4,0 & $20 \%$ & 154 \\
\hline
\end{tabular}

Os parâmetros utilizados nesta Tab. Estão identificados na Figura 9, onde \%EN é a porcentagem do tempo em que o arame-eletrodo está na polaridade negativa

Deve-se ressaltar que os parâmetros utilizados nestes ensaios foram adotados em função de pesquisas anteriores [1618] realizadas pelo grupo Laprosolda (Centro para Pesquisa e Desenvolvimento de Processos de Soldagem). Ensaios preliminares foram necessários [19]para selecionar parâmetros apropriados para se atingir dois níveis de corrente: 115 A e 155 A. Neste sentido, as Tab. 9 e 10 apresentam as réplicas realizadas para se demonstrar a repetibilidade (e desta forma, estabilidade) das condições empregadas.

Os resultados obtidos pelo calorímetro de N2L para estes processos derivativos são mostrados na Tab. 12. É possível observar que maiores valores de potência elétrica (e energia de soldagem por consequência) são atingidos para os processos GMAW-P e GMAW-PV (Fig. 10). Isto é devido ao fato de que estes processos utilizam transferência metálica controlada em voo livre, diferentemente dos demais (STT, RMD e CMT) que utilizam transferência metálica controlada por curto-circuito. Assim, por utilizarem transferência controlada em voo livre, estes processos

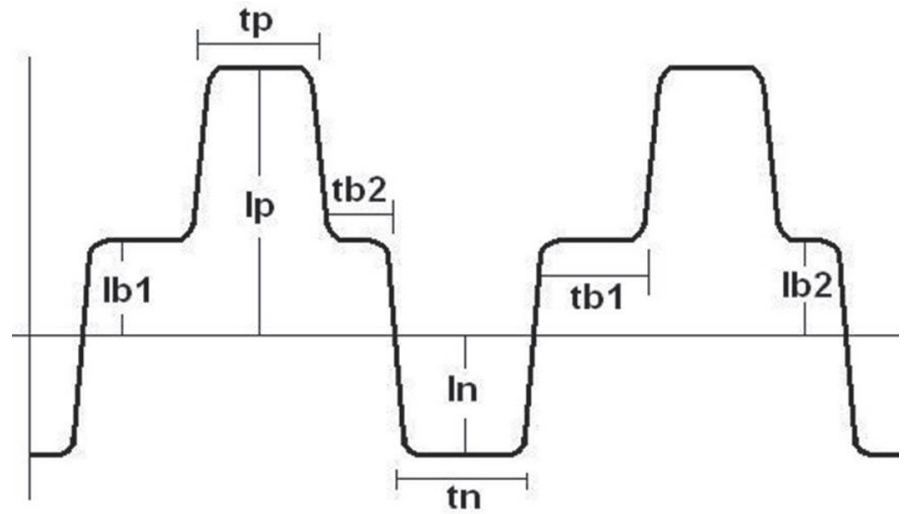

Figura 9. Formato de onda utilizado para o processo GMAWPV [18]

demandam maiores arcos de soldagem e, desta forma, maiores tensões de soldagem. 
Tabela 12. Resultados advindos pelo calorímetro de N2L para os processos derivativos GMAW

\begin{tabular}{|c|c|c|c|c|c|c|c|c|c|c|}
\hline \multirow{5}{*}{ STT } & $\mathrm{I}[\mathrm{A}]$ & $\mathrm{V}_{\mathrm{a}}[\mathrm{cm} / \mathrm{min}]$ & $\mathrm{V}_{\mathrm{s}}[\mathrm{cm} / \mathrm{min}]$ & $\mathrm{L}_{\mathrm{c}}[\mathrm{cm}]$ & $\mathrm{U}_{\mathrm{m}}[\mathrm{V}]$ & $\mathrm{I}_{\mathrm{m}}[\mathrm{A}]$ & $\mathrm{P}_{\text {inst }}[\mathrm{W}]$ & $\mathrm{E}_{\text {sold }}[\mathrm{J} / \mathrm{mm}]$ & $\mathrm{E}_{\mathrm{imp}}[\mathrm{J} / \mathrm{mm}]$ & $\eta_{\text {term }}[\%]$ \\
\hline & 115 & 2,3 & 12,3 & 15 & 18,4 & 119 & 2147 & 1055 & 774 & 73,4 \\
\hline & 115 & 2,3 & 12,3 & 15 & 18,4 & 119 & 2134 & 1045 & 780 & 74,7 \\
\hline & 155 & 3,3 & 17,6 & 15 & 16,6 & 154 & 2606 & 851 & 681 & 79,9 \\
\hline & 155 & 3,3 & 17,6 & 15 & 16,6 & 154 & 2629 & 892 & 692 & 77,5 \\
\hline
\end{tabular}

\begin{tabular}{|c|c|c|c|c|c|c|c|c|c|c|}
\hline \multirow{5}{*}{ RMD } & $\mathrm{I}[\mathrm{A}]$ & $\mathrm{V}_{\mathrm{a}}[\mathrm{cm} / \mathrm{min}]$ & $\mathrm{V}_{\mathrm{s}}[\mathrm{cm} / \mathrm{min}]$ & $\mathrm{L}_{\mathrm{c}}[\mathrm{cm}]$ & $\mathrm{U}_{\mathrm{m}}[\mathrm{V}]$ & $\mathrm{I}_{\mathrm{m}}[\mathrm{A}]$ & $\mathrm{P}_{\text {inst }}[\mathrm{W}]$ & $\mathrm{E}_{\text {sold }}[\mathrm{J} / \mathrm{mm}]$ & $\mathrm{E}_{\mathrm{imp}}[\mathrm{J} / \mathrm{mm}]$ & $\eta_{\text {term }}[\%]$ \\
\hline & 115 & 2,3 & 12,3 & 15 & 17,5 & 118 & 1906 & 903 & 741 & 82,0 \\
\hline & 115 & 2,3 & 12,3 & 15 & 17,5 & 118 & 1917 & 902 & 722 & 80,1 \\
\hline & 155 & 3,3 & 17,6 & 15 & 17,5 & 156 & 2651 & 957 & 730 & 76,3 \\
\hline & 155 & 3,3 & 17,6 & 15 & 17,6 & 154 & 2645 & 944 & 720 & 76,2 \\
\hline & & & & & & & & & & 78,6 \\
\hline \multirow{5}{*}{ CMT } & $\mathrm{I}[\mathrm{A}]$ & $\mathrm{V}_{\mathrm{a}}[\mathrm{cm} / \mathrm{min}]$ & $\mathrm{V}_{\mathrm{s}}[\mathrm{cm} / \mathrm{min}]$ & $\mathrm{L}_{\mathrm{c}}[\mathrm{cm}]$ & $\mathrm{U}_{\mathrm{m}}[\mathrm{V}]$ & $\mathrm{I}_{\mathrm{m}}[\mathrm{A}]$ & $\mathrm{P}_{\text {inst }}[\mathrm{W}]$ & $\mathrm{E}_{\text {sold }}[\mathrm{J} / \mathrm{mm}]$ & $\mathrm{E}_{\mathrm{imp}}[\mathrm{J} / \mathrm{mm}]$ & $\eta_{\text {term }}[\%]$ \\
\hline & 115 & 2,2 & 11,2 & 15 & 10,7 & 114 & 1646 & 861 & 649 & 75,4 \\
\hline & 115 & 2,2 & 11,2 & 15 & 10,9 & 113 & 1658 & 863 & 652 & 75,6 \\
\hline & 155 & 3,3 & 17,0 & 15 & 12,0 & 156 & 2464 & 816 & 622 & 76,3 \\
\hline & 155 & 3,3 & 17,0 & 15 & 11,9 & 157 & 2465 & 851 & 657 & 77,2 \\
\hline & & & & & & & & & & 76,1 \\
\hline \multirow{5}{*}{ GMAW-P } & $\mathrm{I}[\mathrm{A}]$ & $\mathrm{V}_{\mathrm{a}}[\mathrm{cm} / \mathrm{min}]$ & $\mathrm{V}_{\mathrm{s}}[\mathrm{cm} / \mathrm{min}]$ & $\mathrm{L}_{\mathrm{c}}[\mathrm{cm}]$ & $\mathrm{U}_{\mathrm{m}}[\mathrm{V}]$ & $\mathrm{I}_{\mathrm{m}}[\mathrm{A}]$ & $\mathrm{P}_{\text {inst }}[\mathrm{W}]$ & $\mathrm{E}_{\text {sold }}[\mathrm{J} / \mathrm{mm}]$ & $\mathrm{E}_{\mathrm{imp}}[\mathrm{J} / \mathrm{mm}]$ & $\eta_{\text {term }}[\%]$ \\
\hline & 115 & 3 & 15,8 & 15 & 21,2 & 113 & 2773 & 1049 & 744 & 70,9 \\
\hline & 115 & 3 & 15,8 & 15 & 21,5 & 113 & 2816 & 1031 & 725 & 70,3 \\
\hline & 155 & 4 & 21,3 & 15 & 25,7 & 154 & 4368 & 1184 & 813 & 68,7 \\
\hline & 155 & 4 & 21,3 & 15 & 25,3 & 155 & 4360 & 1180 & 812 & 68,9 \\
\hline & & & & & & & & & & 69,7 \\
\hline \multirow{5}{*}{$\begin{array}{c}\text { GMAW- } \\
\text { PV }\end{array}$} & $\mathrm{I}[\mathrm{A}]$ & $\mathrm{V}_{\mathrm{a}}[\mathrm{cm} / \mathrm{min}]$ & $\mathrm{V}_{\mathrm{s}}[\mathrm{cm} / \mathrm{min}]$ & $\mathrm{L}_{\mathrm{c}}[\mathrm{cm}]$ & $\mathrm{U}_{\mathrm{m}}[\mathrm{V}]$ & $\mathrm{I}_{\mathrm{m}}[\mathrm{A}]$ & $\mathrm{P}_{\mathrm{inst}}[\mathrm{W}]$ & $\mathrm{E}_{\text {sold }}[\mathrm{J} / \mathrm{mm}]$ & $\mathrm{E}_{\mathrm{imp}}[\mathrm{J} / \mathrm{mm}]$ & $\eta_{\text {term }}[\%]$ \\
\hline & 115 & 3 & 15,8 & 15 & 21,9 & 115 & 2775 & 1462 & 860 & 58,9 \\
\hline & 115 & 3 & 15,8 & 15 & 22,1 & 115 & 2793 & 1512 & 867 & 57,3 \\
\hline & 155 & 4 & 21,3 & 15 & 23,7 & 154 & 3898 & 1408 & 816 & 57,9 \\
\hline & 155 & 4 & 21,3 & 15 & 23,8 & 154 & 3922 & 1394 & 808 & 57,9 \\
\hline
\end{tabular}

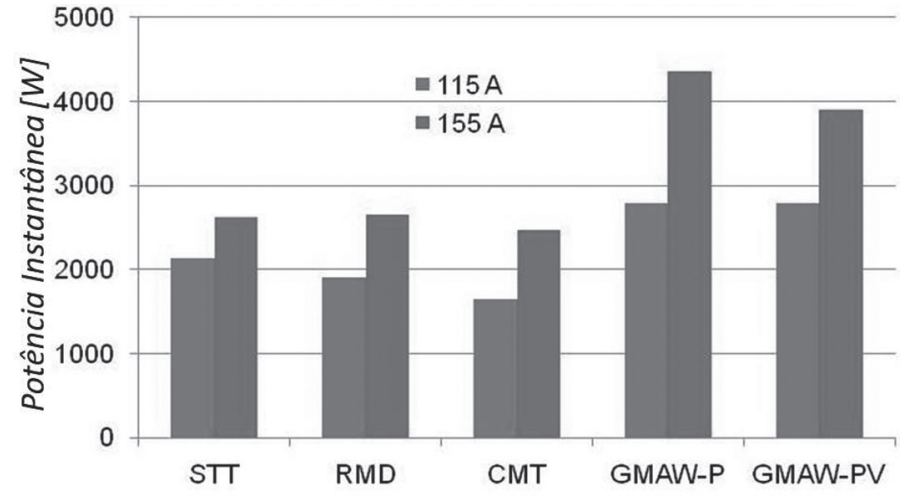

Figura 10. Potência elétrica instantânea para os diferentes processos GMAW investigados
Para o processo STT os valores medidos para rendimento térmico variam entre $74,0 \%$ e $78,7 \%$ quando a corrente varia de $115 \mathrm{~A}$ para $155 \mathrm{~A}$. Isto demonstra as perdas térmicas devido à convecção e radiação do processo. O valor médio de rendimento térmico para o STT foi de $76,4 \%$, o que é comparativamente menor do que $85 \%$ reportado por outros autores $[8,15]$. Para o processo CMT, valores da ordem de $86 \%$ são reportados [8], ao passo que neste trabalho, os valores encontrados foram de $75,4 \%$ para 115 A e $76,8 \%$ para 155 A. A razão para tal discrepância reside no fato de que foram utilizadas faixa paramétricas diferentes, além do fato de que os tempos de soldagens utilizados na literatura [8] foram consideravelmente menores ( 25 segundos na literatura contra $42 \mathrm{~s}$ nestes trabalho).

Durante o processo RMD, rendimentos térmicos de $91,0 \%$ 
e 76,3\% foram medidos respectivamente para correntes de 115 A e 155 A, ou seja, com tendência decrescente similarmente ao já apresentado para os outros processos. Medições de outros autores não foram encontradas na literatura técnico-científica. Neste sentido, acredita-se que, como os valores encontrados estão da ordem daqueles obtidos pelos processos STT e CMT, os resultados obtidos são coerentes.

O principal motivo para que os resultados obtidos para os processos derivativos estejam relativamente próximo devese ao fato de que os parâmetros utilizados foram previamente investigados [16] e representam condições otimizadas para estes processos (STT, RMD e CMT) para uma dada situação (soldagem de tubulação de aço carbono em passe único).

De forma semelhante, o processo GMAW-P apresenta uma queda no rendimento térmico de $70,6 \%$ para $68,8 \%$ quando a corrente aumenta de $115 \mathrm{~A}$ para $155 \mathrm{~A}$, o que indica novamente maiores perdas a maiores correntes e maiores tensões (comprimentos de arco). Estas características também contribuem para maior energia de soldagem. Os valores de rendimento térmico para o GMAW-P encontrado na literatura [20-22] varia entre $62 \%$ a $73 \%$, ao passo que neste trabalho é de $69,7 \%$. Por outro lado, Bosworth [23] reporta eficiências da ordem de $75 \%$ a $80 \%$.

Dentre os processos derivativos investigados, o processo GMAW-PV foi aquele que apresentou menores rendimentos térmicos, da ordem de $58,0 \%$, sem variação estatística entre os níveis de corrente avaliados (115 e 155 A). Na Ref. [24] é reportado que o aporte térmico é da ordem de $25 \%$ menor do que aquele comparado à soldagem GMAW-P e quase metade daquele atingido pela transferência metálica goticular no processo GMAW convencional para uma mesma velocidade de alimentação. Durante o tempo em que o arame-eletrodo está na polaridade positiva, o arco é diretamente conectado à gota em formação e a troca térmica entre o arco e a gota é alta, aquecendo a gota e contribuindo para melhor eficiência térmica quando ao final a gota for transferida. Durante o tempo em que o arame-eletrodo está na polaridade negativa, o arco é conectado à extensão sólida do arame e, embora haja maior taxa de fusão, a área de contato é maior e consequentemente a densidade de corrente é menor. Isto contribui para menor troca térmica entre gota em formação e arco, levando a um menor rendimento térmico. Além disto, o comprimento do arco é maior durante a fase negativa e, como mencionado para os outros processos, quanto maior o comprimento do arco, menor será o rendimento térmico.

\subsection{Comparação entre calorímetro de N2L e fluxo contínuo (água)}

Finalmente é importante comparar os resultados obtidos pelo calorímetro de N2L proposto com outra técnica experimental mais tradicionalmente empregada. Neste caso, o calorímetro de fluxo contínuo (água) foi escolhido. No calorímetro de fluxo contínuo, um fluxo de água constante é mantido sob a chapa soldada e as temperaturas de entrada e saída da água são medidas com uso de termopares, como mostrado na Fig. 11. É também importante mensurar o fluxo real de água caso haja variação no mesmo. Neste trabalho, utilizou-se o sensor SFL 1220 R10. Uma melhor descrição da bancada utilizada é apresentada na Ref. [19] e a Fig. 12 apresenta uma visão geral desta bancada desenvolvida para medição do aporte térmico via calorimetria de fluxo contínuo (água).

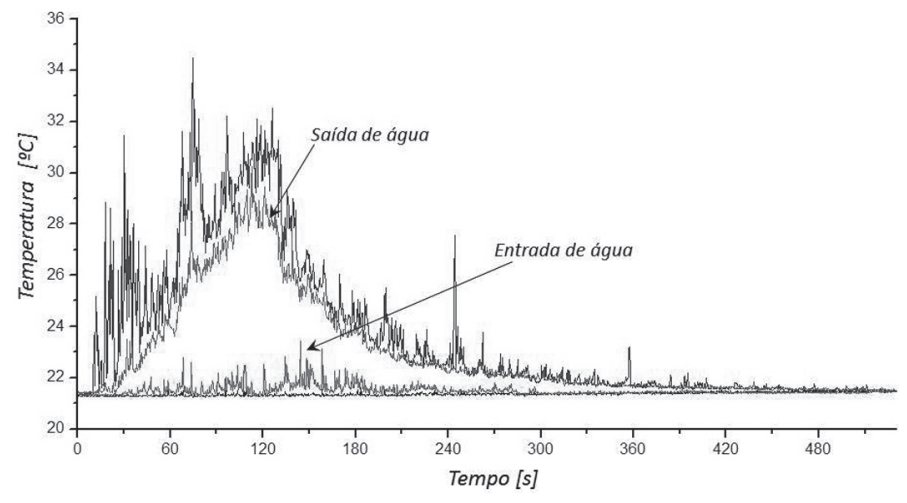

Figura 11. Exemplo de temperaturas medidas no calorímetro de fluxo contínuo (água)

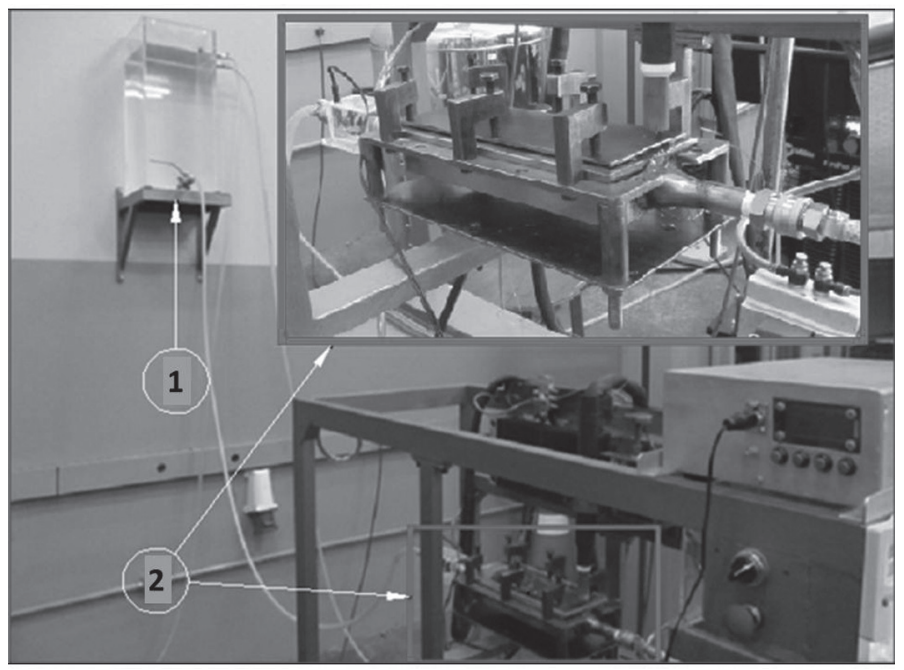

Figura 12. Calorímetro de fluxo contínuo: 1: Recipiente de água para manter pressão constante (coluna d'água) e 2: fixação do calorímetro na bancada experimental

A comparação proposta neste trabalho para ambos os calorímetros será realizada com base nos resultados dos processos GTAW, GMAW convencional por curto-circuito e GMAW convencional goticular. A Tab. 13 apresenta uma seleção dos resultados obtidos para o calorímetro de N2L apresentados anteriormente e se traduzem em valores máximos e mínimos obtidos até aqui para o rendimento térmico. Os mesmos parâmetros utilizados durante a calorimetria por N2L foram também utilizados na calorimetria por fluxo contínuo como apresentado na Tab. 14. A utilização de parâmetros que levem a valores máximos e mínimos de rendimento térmico deve-se ao fato de buscar uma situação que se destaque eventuais diferenças entre os dois calorímetros desenvolvidos.

Graficamente a Fig. 13 apresenta a comparação entre os resultados obtidos para ambos os calorímetros. As diferenças observadas foram de $13,9 \%$ para o GTAW, 10,4\% para o 
Tabela 13. Maiores e menores rendimentos térmicos observados para o calorímetro de N2L

\begin{tabular}{|c|c|c|c|c|c|c|}
\cline { 2 - 7 } \multicolumn{1}{c|}{} & $\mathrm{U}_{\mathrm{m}}[\mathrm{V}]$ & $\mathrm{I}_{\mathrm{m}}[\mathrm{A}]$ & $\mathrm{P}_{\text {inst }}[\mathrm{W}]$ & $\mathrm{E}_{\text {sold }}[\mathrm{J} / \mathrm{mm}]$ & $\mathrm{E}_{\text {imp }}[\mathrm{J} / \mathrm{mm}]$ & Rend $[\%]$ \\
\hline \multirow{2}{*}{ GTAW } & 10,8 & 102 & 1094 & 328 & 259 & 78,9 \\
\cline { 2 - 7 } & 11,9 & 150 & 1785 & 714 & 435 & 60,9 \\
\hline \multirow{2}{*}{ GMAW curto-circuito } & 19,5 & 122 & 2480 & 496 & 407 & 82,0 \\
\cline { 2 - 7 } & 19,5 & 123 & 2495 & 374 & 286 & 76,0 \\
\hline \multirow{2}{*}{ GMAW goticular } & 27,6 & 286 & 7985 & 1597 & 1258 & 78,8 \\
\cline { 2 - 7 } & 28,5 & 247 & 7039 & 1408 & 931 & 66,2 \\
\hline
\end{tabular}

Tabela 14. Resultados obtidos pelo calorímetro de fluxo contínuo (água) com o mesmo conjunto de parâmetros da Tab. 13

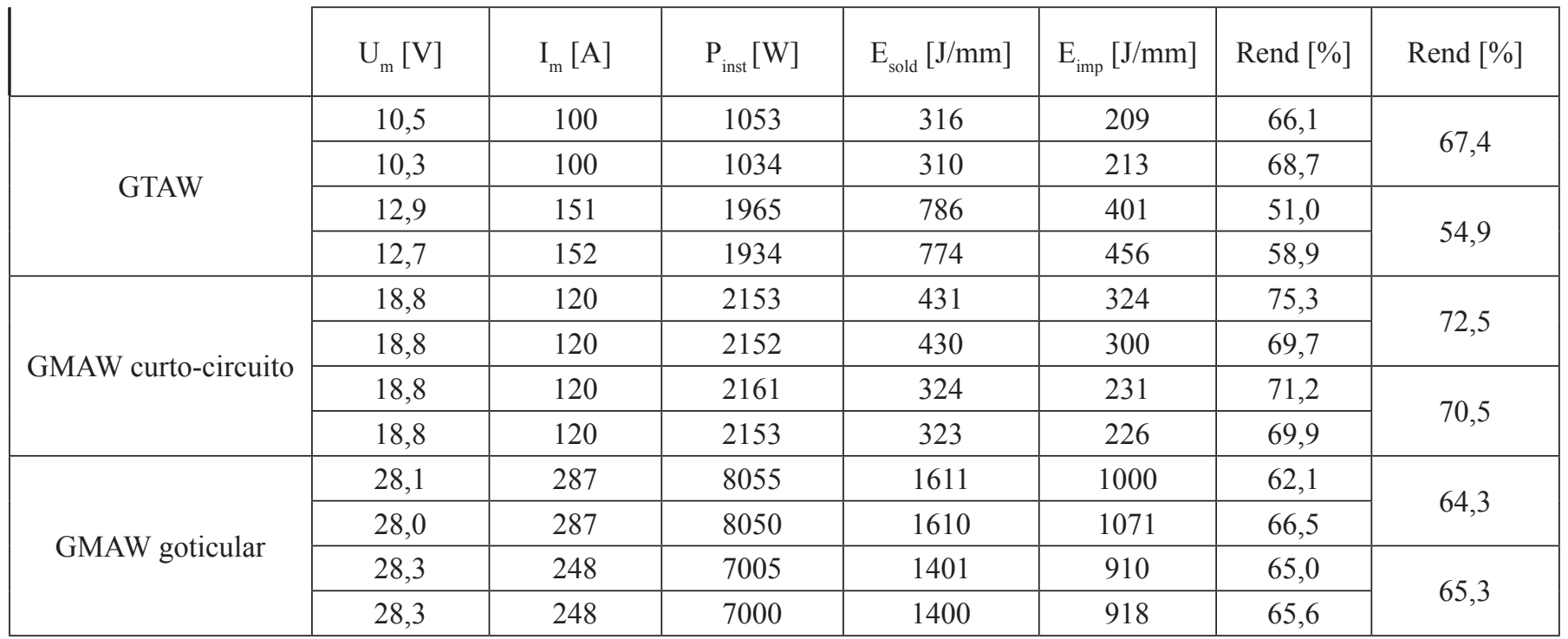

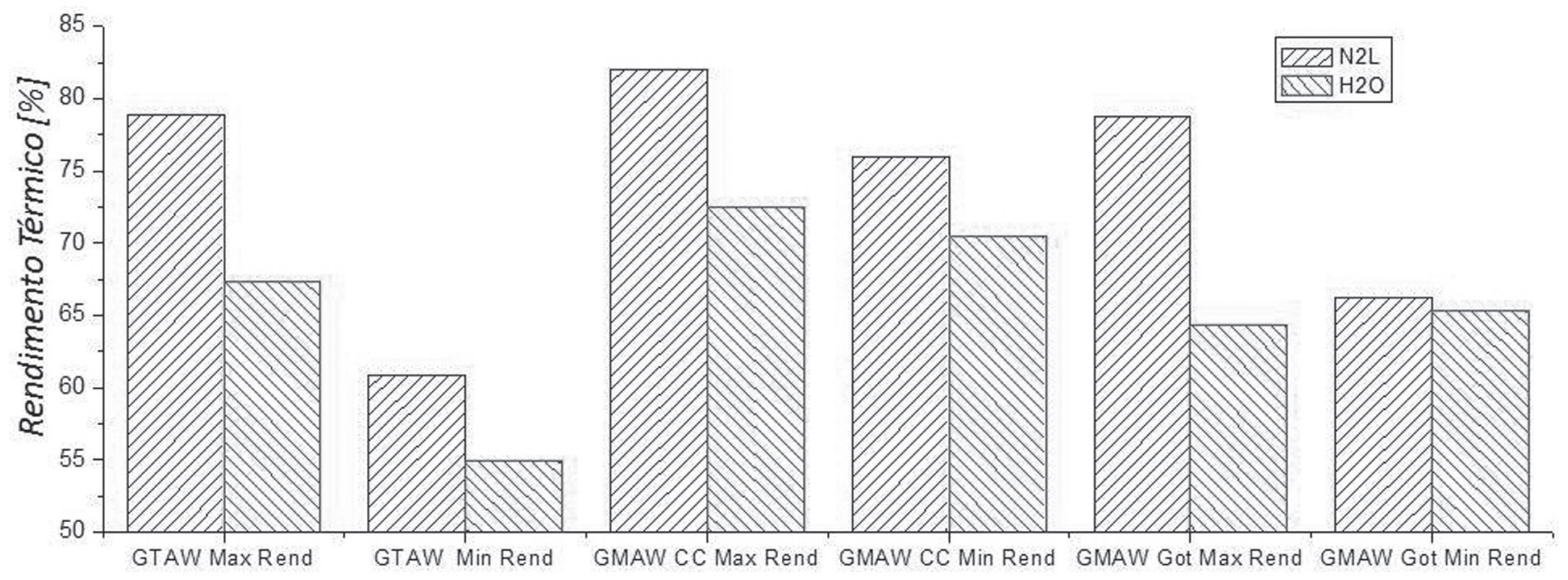

Figura 13. Comparação entre os rendimentos térmicos atingidos por ambos os calorímetros 
GMAW convencional por curto-circuito e 12,0\% para o GMAW convencional goticular. Estas dispersões de resultados são consistentemente menores do que reportados por outros autores [9]. Os menores valores obtidos pelo calorímetro de fluxo contínuo podem ser atribuídos a perdas térmicas do suporte do calorímetro, evaporação da água e formação de camada gasosa, uma vez que para garantir um fluxo laminar, uma baixa vazão de 2,3 $\mathrm{L} / \mathrm{min}$ foi utilizada. Desta forma, acredita-se que os resultados obtidos comprovam o sucesso das técnicas desenvolvidas, em especial do calorímetro de N2L.

\section{Conclusões}

A partir dos resultados obtidos é possível concluir que:

- O equipamento apresenta bom comportamento mecânico e repetibilidade para diferentes espessuras de placa teste não sendo superior a $1,5 \%$ de diferença entre os resultados;

- No processo TIG para um mesmo comprimento de arco e velocidade de soldagem se observou que o acréscimo da corrente influencia de forma negativa o rendimento térmico do processo;

- Para o processo TIG o aumento do comprimento do arco (3 a $5 \mathrm{~mm}$ ) representou uma diminuição do rendimento térmico de $5 \%$ aproximadamente;

- Para os parâmetros propostos na avaliação do processo TIG o aumento da velocidade de soldagem aumenta o rendimento térmico do processo, diminuindo-se as perdas por convecção e radiação para o entorno;

- Os valores de rendimento térmico encontrados neste trabalho para o processo TIG concordam com os resultados encontrados na literatura para condições semelhantes de soldagem.

- Durante a soldagem GMAW convencional, a transferência por curto-circuito levou a maiores rendimentos térmicos do processo quando comparado à transferência goticular;

- Os processos GMAW derivativos podem ser divididos em dois grupos: um que utiliza transferência controlada por curto-circuito (STT, RMD e CMT) e outro que utiliza transferência controlada por voo livre (GMAW-P e GMAW$\mathrm{PV})$. O rendimento térmico do primeiro grupo é maior do que o aquele observado para o segundo;

- O calorímetro de fluxo contínuo (água) desenvolvido mediu rendimentos térmicos menores do que aqueles aferidos pelo calorímetro de N2L. Entretanto, a dispersão de dados ficou menor do que aquele apresentado na literatura técnico-científica, o que comprova o sucesso das técnicas desenvolvidas, em especial do calorímetro de N2L.

\section{Agradecimentos}

Os autores agradecem à Fapemig (Processo TEC - PPM00511-12) e ao CNPq (Processo 307710/2011-4).

\section{Referências Bibliográficas}

[1] ROSENTHAL, D. Mathematical Theory of Heat Distribution During Welding and Cutting. Welding Journal, 20 (5), 220s-234s, 1941.

[2] NILES, R., \& JACKSON, C. E. Weld Thermal Efficiency of the TIG Process. Welding Journa, 25s-32s, 1985.

[3] DUTTA, P. et. al. Determination of Gas Tunsgten Arc Welding Efficiencie. Experimental Thermal and Fluid Science (9), 80-89, 1994.

[4] LU, J., e KOU, S. Power Inputs in Gas Metal Arc Welding of Aluminum - Part 2. Welding Journal, 60 (11), 452s-456s, 1989.

[5] GIEDT, W. H. et al. Welding Efficiency: Calorimetric and Temperature Field Measurements. Welding Journal , 68 (1), 28s-32s, 1989.

[6] CANTIN, G. M., \& FRANCIS, J. A. Arc Power and Efficiency in Gas Tungsten Arc Welding of Aluminium. Science and Technology of welding and joining , 10 (2), 200-210, 2005. [7] PEPE, N. Advances in Metal Arc Welding and Application to Corrosion Resistant Alloy Pipes. Cranfield, UK: Cranfield University, 2010.

[8] PEPE, N. et. al. Measuring the process efficiency of controlled gas metal arc welding processes. Science andTechnology of Welding and Joing , 412-417, 2011.

[9] ZIJP, J. Heat transport during gas tungsten arc welding. Maandag: Technische Universiteit Delft, 1990.

[10] MEACKER, H. H., and STABLEIN, H. G. What Keeps an arc Standing in a Croos FLow? IEEE. Trans. Plasma Sci, 291299, 1986.

[11] VILARINHO, L. O. O arco TIG a 100 A e Argônio Puro é um Arco Especial? Soldagem e Inspeção , 14, 353-357, 2009.

[12] SMARTT, H. et. al. Heat Transfer in Gas Tungsten Arc Welding. International Welding Congress. Toronto, Canada, 1985.

[13] FUERSHBACH, P. K. (1991). A Study of Melting Efficiency in Plasma Arc and Tungsten Arc Welding. Welding Journal , 287s-297s.

[14] DUPONT, J. N., and MARDER, A. R. Thermal Efficiency of Arc Welding Processes. Department of Material Science and Engineering, 406s-416s, 1995.

[15] HSU, C.; SOLTIS, E. P. Heat Input Comparison of STT vs. Short-Circuiting and Pulsed GMAW vs. CV Processes. 6th International Trends in Welding Resaerch Conference Proceedings. Pine Mountain, GA, 2003. 369-374.

[16] COSTA, T.F. Aplicação de Processos MAG Curto-circuito Convencional e Controlado na Soldagem de Dutos de Aço Carbono. 2011. 197 f. Dissertação de mestrado, Universidade Federal de Uberlândia.

[17] OJO, E. And SCOTTI, A. Determinação dos parârametros de pulso na soldagem MIG/MAG pulsada- Parte I: Arame ER70S-6 de 1,2 mm e gás $\mathrm{Ar}+5 \% \mathrm{O}_{2}$. Uberlândia, $\mathrm{MG}$, Brasil: LAPROSOLDA/UFU 24, 2005.

[18] VILARINHO, L.O. et al. Methodology for Parameter Calculation of VP-GMAW. Welding Journal, April, p.92s-98s, 2009. 
[19] AREVAlO, H. H. Desenvolvimento e Avaliação de Calorímetros Via Nitrogênio Liquido e Fluxo Continuo (Água) para Processos de Soldagem. 2011. 145p. Dissertação de Mestrado, Universidade Federal de Uberlândia - MG, Brasil.

[20] ALLUM, C.J.; QUINTINO, L. Control of Fusion Characteristics in Pulsed Current MIG Welding - Part 2. Metal Construction. 17 (1985): 21-24.

[21] KENNEY, K. L.; MILLER, K. S.; SMARTT, E H. B. Heat Transfer in Pulsed Gas Metal Arc Welding. Fifth International Conference on Trends in Welding Research. Pine Mountain, Georgia, USA. 1998. 355-361.

[22] JOSEPH, A., et al. Measurement and Calculation of Arc Power and Heat Transfer Efficiency in Pulsed Gas Metal Arc Welding. Science and Technology of Welding and Joining, 8, $\mathrm{n}$. 6 (2003): 400-406.

[23] BOSWORTH, M. Effective Heat Input in Pulsed Current Gas Metal Arc Welding with Solid Wire Electrodes. Welding Journal , 70, 1991: 111s-117s.

[24] HARWIG, D.D. Arc Behavior and Metal Transfer in the VP-GMAW Process. Cranfield University: School on Industrial Manufacturing Science, 2003. 